\title{
Deformation concentration for martensitic microstructures in the limit of low volume fraction
}

\author{
December 22, 2015 \\ Sergio Conti, Johannes Diermeier, and Barbara Zwicknagl \\ Institut für Angewandte Mathematik, Universität Bonn \\ 53115 Bonn, Germany
}

\begin{abstract}
We consider a singularly-perturbed nonconvex energy functional which arises in the study of microstructures in shape memory alloys. The scaling law for the minimal energy predicts a transition from a parameter regime in which uniform structures are favored, to a regime in which the formation of fine patterns is expected. We focus on the transition regime and derive the reduced model in the sense of $\Gamma$-convergence. The limit functional turns out to be similar to the Mumford-Shah functional with additional constraints on the jump set of admissible functions. One key ingredient in the proof is an approximation result for $S B V^{p}$ functions whose jump sets have a prescribed orientation.
\end{abstract}

\section{Introduction}

In this paper, we consider for $\theta \in(0,1 / 2], \varepsilon>0$ and $p \in(1, \infty)$ the energy functional $I_{\varepsilon, \theta}^{p}: \mathcal{B} \rightarrow[0, \infty)$,

$$
I_{\varepsilon, \theta}^{p}(v):=\int_{(0,1)^{2}}\left|\partial_{1} v\right|^{p}+\min \left\{\left|\partial_{2} v+\theta\right|^{p},\left|\partial_{2} v-(1-\theta)\right|^{p}\right\} \mathrm{d} x+\varepsilon\left|D^{2} v\right|\left((0,1)^{2}\right),
$$

where

$$
\mathcal{B}:=\left\{v \in W^{1, p}\left((0,1)^{2}\right): \partial_{1} v, \partial_{2} v \in B V\left((0,1)^{2}\right), v(0, \cdot)=0\right\} .
$$

Here we use the short-hand notation $\partial_{j} v:=\frac{\partial}{\partial x_{j}} v$ for $j=1,2$. It turns out that for fixed $p$, there are two scaling regimes for the minimal energy. If the coefficient $\varepsilon$ of the higher-order term is large, on a scale set by $\theta$, then low-energy maps $v$ are approximately constant, and the optimal energy is of order $\theta^{p}$. If instead $\varepsilon$ is very small fine structures arise, with $\partial_{2} v$ oscillating between $-\theta$ and $1-\theta$, on a scale which refines close to the $\left\{x_{1}=0\right\}$ boundary. Precisely, we have the following result.

Theorem 1.1. For any $p \in(1, \infty)$ there exists $c>0$ such that for all $\theta \in(0,1 / 2]$ and all $\varepsilon>0$,

$$
\frac{1}{c} \theta^{p} \min \left\{1,\left(\varepsilon / \theta^{p}\right)^{p /(p+1)}\right\} \leq \inf _{\mathcal{B}} I_{\theta, \varepsilon}^{p} \leq c \theta^{p} \min \left\{1,\left(\varepsilon / \theta^{p}\right)^{p /(p+1)}\right\} .
$$


The proof of this scaling law (1.3) is fairly standard and well known in the case $p=2$ (see $[35,36,16,23,45])$, and we provide it in the appendix for general $p$. In this paper, we focus on the transition regime, in which $\varepsilon / \theta^{p}=: \sigma \in(0, \infty)$ is fixed. In this case, $\inf I_{\sigma \theta^{p}, \theta}^{p} \sim \theta^{p}$, and, after rescaling $v\left(x_{1}, x_{2}\right):=\theta\left(u\left(x_{1}, x_{2}\right)-x_{2}\right)$ and $E_{\sigma, \theta}^{p}(u):=I_{\sigma \theta^{p}, \theta}^{p}(v) / \theta^{p}$, we are left to study

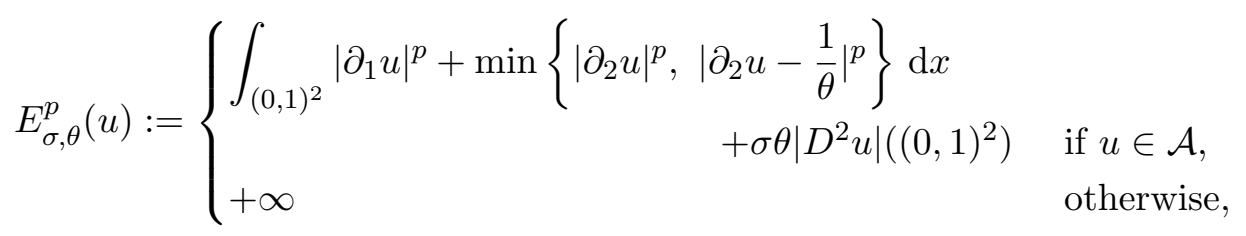

where

$$
\mathcal{A}:=\left\{u \in W^{1, p}\left((0,1)^{2}\right): \partial_{1} u, \partial_{2} u \in B V\left((0,1)^{2}\right), u\left(0, x_{2}\right)=x_{2}\right\} .
$$

For small $\theta$ the deformation concentrates on a set of small volume. In particular, $\partial_{2} u$ becomes of order $1 / \theta$ in a small region of order $\theta$. The length of the boundary of this exceptional set is controlled by the second-order term, since in going across it the gradient $D u$ has two jumps of order $1 / \theta$. Asymptotically $u$ approaches locally an $S B V$ function, with the additional property that the singular part of the gradient has a specific orientation.

Our main result is the $\Gamma$-limit of $E_{\sigma, \theta}^{p}$ as $\theta \rightarrow 0$. Precisely, we set

$$
E_{\sigma}^{p}(u):= \begin{cases}\int_{(0,1)^{2}}\left(\left|\partial_{1} u\right|^{p}+\left|\partial_{2} u\right|^{p}\right) \mathrm{d} x+2 \sigma \mathcal{H}^{1}\left(J_{u}\right) & \text { if } u \in \overline{S B V}_{y}^{p} \\ +\infty & \text { otherwise }\end{cases}
$$

where

$$
\begin{aligned}
\overline{S B V}_{y}^{p}:=\left\{u \in S B V_{\mathrm{loc}}\left((0,1)^{2}\right):\right. & \nabla u \in L^{p}\left((0,1)^{2} ; \mathbb{R}^{2}\right), u\left(0, x_{2}\right)=x_{2}, \\
& \text { and } \left.[u] \nu_{u} \in[0, \infty) e_{2} \mathcal{H}^{1} \text { - a.e. }\right\} .
\end{aligned}
$$

We remark that for any $u \in \overline{S B V}_{y}^{p}$ one has $|D u|((0,1) \times(\delta, 1-\delta))<\infty$ for all $\delta \in(0,1 / 2)$, see Lemma 3.3 below. We prove the following theorem.

Theorem 1.2. (i) Compactness. Suppose that $\theta_{k} \rightarrow 0$, and let $u_{k} \in \mathcal{A}$ such that $E_{\sigma, \theta_{k}}^{p}\left(u_{k}\right) \leq M$ for some $M>0$. Then there exists a subsequence (not relabeled) and $u \in \overline{S B V}_{y}^{p}$ such that $u_{k} \rightarrow u$ in $L^{1}\left((0,1)^{2}\right)$ and $u_{k} \stackrel{*}{\rightarrow} u$ in $B V((0,1) \times(\delta, 1-\delta))$ for all $\delta \in(0,1 / 2)$.

(ii) Lower bound. Suppose that $\theta_{k} \rightarrow 0$. Let $u_{k} \in \mathcal{A}$ and assume that $u_{k} \rightarrow u$ in $L^{1}\left((0,1)^{2}\right)$ for some $u \in \overline{S B V}_{y}^{p}$. Then $E_{\sigma}^{p}(u) \leq \liminf _{k \rightarrow \infty} E_{\sigma \theta_{k}^{p}, \theta_{k}}^{p}\left(u_{k}\right)$.

(iii) Upper bound. Let $\theta_{k} \rightarrow 0$ and $u \in \overline{S B V}_{y}^{p}$. Then there exist $u_{k} \in \mathcal{A}$ such that $u_{k} \rightarrow u$ in $L^{1}\left((0,1)^{2}\right)$ and $E_{\sigma}^{p}(u)=\lim \sup _{k \rightarrow \infty} E_{\sigma \theta_{k}^{p}, \theta_{k}}^{p}\left(u_{k}\right)$. 
By (i) the sequence in (iii) obeys also $u_{k} \stackrel{*}{\rightarrow} u$ in $B V((0,1) \times(\delta, 1-\delta))$ for all $\delta \in(0,1 / 2)$. The result of Theorem 1.2 has been announced in [25] for the case $p=2$ and is part of Johannes Diermeier's Ph.D. thesis (see [26]). We focus here on the two-dimensional situation, and refer to [26] for an analogous result in one dimension. For ease of notation, we consider the functional on the unit square $(0,1)^{2}$, but we point out that our analysis can be easily adapted to treat more general domains $\Omega \subset \mathbb{R}^{2}$.

The limit functional $E_{\sigma}^{p}$ bears similarities with the Mumford-Shah functional (see $[21,38]$ ). The main difference lies in the constraints on the jump sets of admissible functions, see (1.7). This introduces technical difficulties in the proof of the upper bound, for which we follow the general strategy to use density of functions with a simpler structure. More precisely, we provide explicit constructions of recovery sequences only for functions whose jump sets consist of finitely many segments and which are smooth away from their jump sets, and prove an accompanying density result to deal with general functions in $\overline{S B V}_{y}^{p}$. To the best of our knowledge, there are no approximation results in the literature that respect the constraints for functions in $\overline{S B V}_{y}^{p}$ (see e.g. $[19,22]$ and the references given there).

The motivation for our analysis comes from the mathematical study of martensitic phase transitions, where the functional $I_{\theta, \varepsilon}^{2}$ as defined in (1.1) arises in the study of microstructures near interfaces (see $[35,36])$. Here, $(0,1)^{2}$ represents a martensitic region meeting rigid austenite at an interface $\left\{x_{1}=0\right\}$. The first two terms in the definition of $I_{\varepsilon, \theta}^{p}$ model the elastic energy, and the last term can be interpreted as an interfacial energy between different variants of martensite; the coefficient $\varepsilon$ represents a typical interfacial energy per unit length. The preferred gradients $(0,-\theta)^{T}$ and $(0,1-\theta)^{T}$ correspond to two variants of martensite, and the parameter $\theta \in(0,1 / 2]$ measures compatibility between the austenite and the martensite phases: For $\theta=0$, austenite and martensite are compatible in the sense that $v_{c}:=0$ satisfies $I_{\varepsilon, \theta}^{p}\left(v_{c}\right)=\min I_{\varepsilon, \theta}^{p}=0$, while for $\theta>0$, we have $\inf I_{\varepsilon, \theta}^{p}>0$. It has been found in experiments that compatibility between the phases is closely related to the width of the hysteresis loop accompanying the phase transformation (see $[29,20,43,42,37,40,2])$. This theory of hysteresis predicts that the energy barrier inf $I_{\varepsilon, \theta}^{p}$ plays a major role for reversibility of the phase transformation (see $[44,11])$.

As pointed out before, the scaling law for the minimal energy (1.3) is wellunderstood for the physically relevant case $p=2$ (see [35, 36, 16, 23, 45, 18]). These studies suggest a transition between uniform structures and the formation of microstructures. Some of the results have been extended also to the vectorvalued case (see $[8,9,10,24,7,6,32,33]$ ). Similar phenomena have been found for a variety of variational models, with applications including pattern formation in ferromagnets (see $[15,13,39,34]$ ), in type-1-superconductors (see $[14,12,17]$ ), and in thin compressed films (see $[4,30,31,5,3]$ ). In many situations, peculiar structures arise in the limit of small volume fraction, which corresponds to the 
(a)

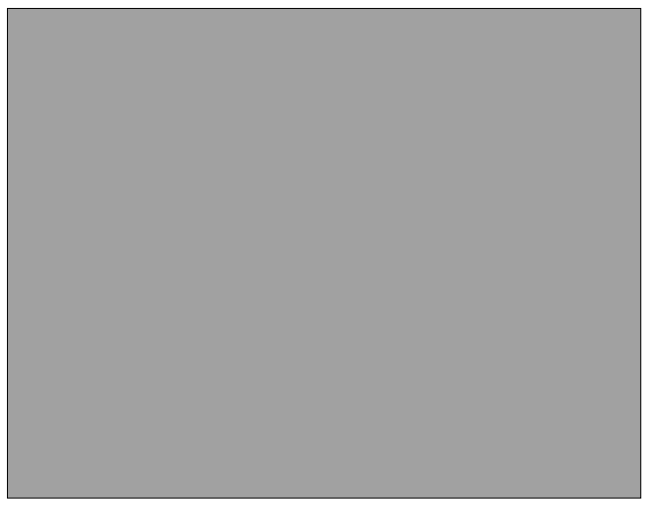

(b)

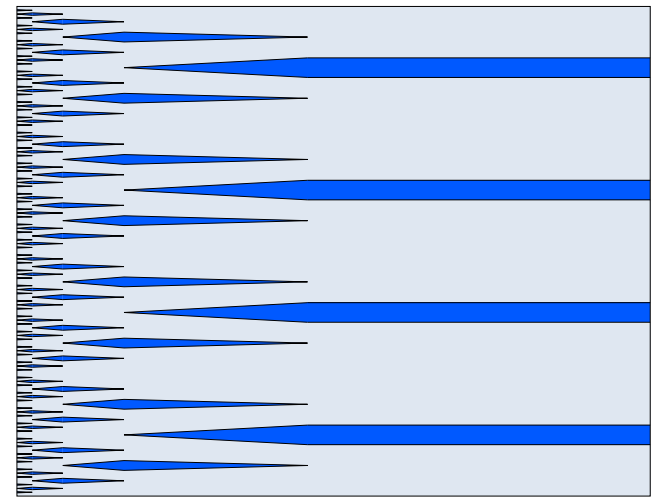

Figure 1: (a) uniform test function $v_{c}=0$. (b) sketch of the branching construction $v_{b}$. The interface to austenite is at the left edge. Different colors indicate different values of the order parameter $\partial_{2} v$.

limit $\theta \rightarrow 0$ considered in this paper [14, 12, 23, 45, 18].

Typical test functions that can be used to prove the second inequality in (1.3) are sketched in Figure 1. On the one hand, the constant function $v_{c}:=0$ satisfies $I_{\theta, \varepsilon}^{p}\left(v_{c}\right)=\theta^{p}$. On the other hand, if $\varepsilon \leq \theta^{p}$, then a self-similarly refining function $v_{b}$ as introduced in [36, Lemma 2.1] yields $I_{\theta, \varepsilon}^{p}\left(v_{b}\right) \leq c \theta^{p}\left(\varepsilon / \theta^{p}\right)^{p /(p+1)}$ (see appendix for a precise definition). For low-hysteresis shape memory alloys, one expects both parameters $\theta$ and $\varepsilon$ to be small. If one of them is much smaller than the other, then one of the two mentioned regimes is expected to dominate the picture, as indicated by the scaling in Theorem 1.1. Theorem 1.2 addresses the transition regime $\varepsilon \sim \theta^{p}$ in the asymptotic situation $\varepsilon, \theta \rightarrow 0$, and provides a step towards the better understanding of the onset of microstructures.

The remaining part of the paper is structured as follows. After setting some notation in Section 2, we prove the lower bound and the accompanying compactness result, i.e., parts (i) and (ii) of Theorem 1.2 in Section 3. In Section 4 we prove the upper bound, i.e., part (iii) of Theorem 1.2. This is done in two steps. First, in Subsection 4.1, we provide explicit constructions of recovery sequences for functions whose jump sets are finite unions of segments. Subsequently, in Subsection 4.2 we prove a density result which shows that it suffices to consider those simpler functions.

\section{Notation and basic facts}

The parameters $p \in(1, \infty)$ and $\sigma \in(0, \infty)$ are arbitrary but fixed. We write briefly $E_{\theta}:=E_{\sigma, \theta}^{p}$ and $E:=E_{\sigma}^{p}$. We write $c$ and $C$ to denote generic positive constants that may change from expression to expression. We denote by $e_{1}$ and $e_{2}$ the standard basis vectors in $\mathbb{R}^{2}$. For a measurable set $\Omega \subset \mathbb{R}^{n}$ we denote by $|\Omega|$ its $n$-dimensional Lebesgue measure. We often do not relabel subsequences. 
We briefly collect some definitions and properties we use in this work. For precise definitions, details and proofs we refer to $[1,27]$. Let $\Omega \subset \mathbb{R}^{2}$ be open. For $u \in$ $B V(\Omega)$, we denote the distributional gradient by $D u$, the approximate differential by $\nabla u$, the jump set by $J_{u}$, and the jump function by $[u]$. The jump set $J_{u}$ is countably $\mathcal{H}^{1}$-rectifiable, and we denote the generalized normal by $\nu_{u}: J_{u} \rightarrow S^{1}$. We will use the decomposition $D u=D^{a c} u+D^{J} u+D^{C} u$, where $D^{a c} u=\nabla u \mathcal{L}^{2}$ denotes the absolutely continuous part with respect to the Lebesgue measure $\mathcal{L}^{2}$, $D^{J} u=[u] \nu_{u} \mathcal{H}^{1}\left\llcorner J_{u}\right.$ denotes the jump part and $D^{C} u$ is the Cantor part. We denote the singular part by $D^{S} u:=D^{J} u+D^{C} u$. The space $S B V^{p}(\Omega)$ contains those functions in $B V(\Omega)$ such that the Cantor part vanishes, the approximate differential is in $L^{p}$, and the jump set has finite length.

We will use various slicing arguments (see [1, Section 3.11]). On the one hand, we use that for $u \in B V(\Omega)$, the singular parts $D^{C} u$ and $D^{J} u$ can be obtained from the respective parts of the slices. On the other hand, we use specific properties of slices of admissible functions. In our analysis, the space $\overline{S B V}_{y}^{p}$ as defined in (1.7) plays a crucial role. We point out that for the density part (see Subsection 4.2 ), we work with a slightly different space $S B V_{y}^{p}(\Omega)$ defined on general open sets $\Omega \subset \mathbb{R}^{2}$, i.e.,

$$
S B V_{y}^{p}(\Omega):=\left\{u \in S B V^{p}(\Omega):[u] \nu_{u} \in[0, \infty) e_{2} \mathcal{H}^{1}-\text { a.e. }\right\} .
$$

We remark that the definition of the space $\overline{S B V_{y}^{p}}$ includes the boundary data, whereas functions in $S B V_{y}^{p}\left((0,1)^{2}\right)$ do not need to satisfy them. On the other hand, $S B V_{y}^{p}$ is a subset of $S B V^{p}$, while $\overline{S B V_{y}^{p}}$ is not (see also Lemma 3.3 and Example 3.2 below).

If $u \in S B V_{y}^{p}(\Omega)$, then for $\mathcal{H}^{1}$-almost every $x_{2} \in \mathbb{R}$ the horizontal slice $u^{x_{2}}(t):=$ $u\left(t, x_{2}\right)$ belongs to $W^{1, p}\left(I_{x_{2}}\right)$, where $I_{x_{2}}:=\left\{t:\left(t, x_{2}\right) \in \Omega\right\}$, and its derivative is given by $\left(u^{x_{2}}\right)^{\prime}(t)=\partial_{1} u\left(t, x_{2}\right)$. Correspondingly, for $\mathcal{H}^{1}$-almost every $x_{1} \in \mathbb{R}$ the vertical slice $u^{x_{1}}(s):=u\left(x_{1}, s\right)$ belongs to $S B V^{p}\left(I_{x_{1}}\right)$, where $I_{x_{1}}:=\left\{s:\left(x_{1}, s\right) \in\right.$ $\Omega\}$, with derivative $D u^{x_{1}}=\partial_{2} u \mathcal{L}^{1}+[u] \mathcal{H}^{0}\left\llcorner\left\{s:\left(x_{1}, s\right) \in J_{u}\right\}\right.$.

\section{Compactness and lower bound}

In this section we prove parts (i) and (ii) of Theorem 1.2.

Proposition 3.1. Suppose that $M>0, \theta_{k} \rightarrow 0$ and $u_{k} \in \mathcal{A}$ are such that $E_{\theta_{k}}\left(u_{k}\right) \leq M$ for all $k \in \mathbb{N}$. Then there exist a subsequence and $u \in \overline{S B V}_{y}^{p}$ such that $u_{k} \rightarrow u$ strongly in $L^{1}\left((0,1)^{2}\right), u_{k} \stackrel{*}{\rightarrow} u$ in $B V((0,1) \times(\delta, 1-\delta))$ for all $\delta \in\left(0, \frac{1}{2}\right)$, and $E(u) \leq \liminf _{k \rightarrow \infty} E_{\theta_{k}}\left(u_{k}\right)$.

Proof. Step 1: A priori bounds and identification of $u$. We have

$$
\left\|\partial_{1} u_{k}\right\|_{L^{1}\left((0,1)^{2}\right)} \leq\left\|\partial_{1} u_{k}\right\|_{L^{p}\left((0,1)^{2}\right)} \leq M^{1 / p},
$$


and thus, using $u_{k}\left(0, x_{2}\right)=x_{2}$,

$$
\left\|u_{k}\right\|_{L^{1}\left((0,1)^{2}\right)} \leq\left\|\partial_{1} u_{k}\right\|_{L^{1}\left((0,1)^{2}\right)}+\left\|u_{k}(0, \cdot)\right\|_{L^{1}((0,1))} \leq M^{1 / p}+\frac{1}{2} .
$$

For $\delta \in(0,1 / 2)$ set

$$
A_{k}^{\delta}:=\left\{\left(x_{1}, x_{2}\right) \in(0,1) \times(\delta, 1-\delta):\left|\partial_{2} u_{k}\left(x_{1}, x_{2}\right)\right| \leq\left|\partial_{2} u_{k}\left(x_{1}, x_{2}\right)-\frac{1}{\theta_{k}}\right|\right\}
$$

and $B_{k}^{\delta}:=((0,1) \times(\delta, 1-\delta)) \backslash A_{k}^{\delta}$. Then,

$$
\left\|\left(\partial_{2} u_{k}\right) \chi_{A_{k}^{\delta}}\right\|_{L^{1}\left((0,1)^{2}\right)} \leq\left\|\left(\partial_{2} u_{k}\right) \chi_{A_{k}^{\delta}}\right\|_{L^{p}\left((0,1)^{2}\right)} \leq M^{1 / p},
$$

and similarly, using $\left|\partial_{2} u_{k}\right| \leq\left|\partial_{2} u_{k}-\frac{1}{\theta_{k}}\right|+\frac{1}{\theta_{k}}$, we obtain

$$
\left\|\left(\partial_{2} u_{k}\right) \chi_{B_{k}^{\delta}}\right\|_{L^{1}\left((0,1)^{2}\right)} \leq M^{1 / p}+\frac{1}{\theta_{k}}\left|B_{k}^{\delta}\right| .
$$

Since $\partial_{2} u_{k} \geq \frac{1}{2 \theta_{k}}$ in $B_{k}^{\delta}$, we obtain

$$
\int_{0}^{1}\left(u_{k}\left(x_{1}, 1-\delta\right)-u_{k}\left(x_{1}, \delta\right)\right) \mathrm{d} x_{1}=\int_{B_{k}^{\delta}} \partial_{2} u_{k} \mathrm{~d} x+\int_{A_{k}^{\delta}} \partial_{2} u_{k} \mathrm{~d} x \geq\left|B_{k}^{\delta}\right| \frac{1}{2 \theta_{k}}-M^{1 / p} .
$$

Therefore, for almost every $x_{2} \in(0, \delta)$, we have

$$
\begin{aligned}
& \int_{0}^{1}\left(u_{k}\left(x_{1}, 1-\delta+x_{2}\right)-u_{k}\left(x_{1}, x_{2}\right)\right) \mathrm{d} x_{1} \\
= & \int_{0}^{1}\left(u_{k}\left(x_{1}, 1-\delta\right)-u_{k}\left(x_{1}, \delta\right)+\int_{1-\delta}^{1-\delta+x_{2}} \partial_{2} u_{k}\left(x_{1}, t\right) \mathrm{d} t+\int_{x_{2}}^{\delta} \partial_{2} u_{k}\left(x_{1}, t\right) \mathrm{d} t\right) \mathrm{d} x_{1} \\
\geq & \left|B_{k}^{\delta}\right| \frac{1}{2 \theta_{k}}-M^{1 / p}+\int_{(0,1)^{2}} \min \left\{\partial_{2} u_{k}\left(x_{1}, x_{2}\right), 0\right\} \mathrm{d} x_{1} \geq \frac{1}{2 \theta_{k}}\left|B_{k}^{\delta}\right|-2 M^{1 / p}
\end{aligned}
$$

where in the last step we used (3.3). Hence,

$$
\begin{aligned}
\left\|u_{k}\right\|_{L^{1}\left((0,1)^{2}\right)} & \geq \int_{0}^{1} \int_{0}^{\delta}\left(\left|u_{k}\left(x_{1}, 1-\delta+x_{2}\right)\right|+\left|u_{k}\left(x_{1}, x_{2}\right)\right|\right) \mathrm{d} x_{2} \mathrm{~d} x_{1} \\
& \geq \int_{0}^{1} \int_{0}^{\delta}\left(u_{k}\left(x_{1}, 1-\delta+x_{2}\right)-u_{k}\left(x_{1}, x_{2}\right)\right) \mathrm{d} x_{2} \mathrm{~d} x_{1} \\
& \geq \delta\left(\frac{1}{2 \theta_{k}}\left|B_{k}^{\delta}\right|-2 M^{1 / p}\right),
\end{aligned}
$$

and thus, combining with (3.3), (3.4) and (3.2), we obtain $\left\|\partial_{2} u_{k}\right\|_{L^{1}((0,1) \times(\delta, 1-\delta))} \leq$ $\frac{C}{\delta}\left(M^{1 / p}+1\right)$. Putting things together, we have

$$
\left\|u_{k}\right\|_{W^{1,1}((0,1) \times(\delta, 1-\delta))} \leq \frac{C}{\delta}\left(M^{1 / p}+1\right) .
$$


Therefore, by a diagonal sequence argument, there are a subsequence and a limit function $u:(0,1)^{2} \rightarrow \mathbb{R}$ such that $u_{k} \stackrel{*}{\rightarrow} u$ in $B V((0,1) \times(\delta, 1-\delta))$ for all $\delta \in\left(0, \frac{1}{2}\right)$. Further, $u_{k} \rightarrow u$ strongly in $L^{1}((0,1) \times(\delta, 1-\delta))$ for all $\delta$. The same argument in (3.2) shows that $\left\|u_{k}\right\|_{L^{1}((0,1) \times((0, \delta) \cup(1-\delta, 1)))} \leq M^{1 / p} \delta^{1 / p^{\prime}}+2 \delta$. Therefore $u_{k} \rightarrow u$ strongly in $L^{1}\left((0,1)^{2}\right)$.

Step 2: Show that $u \in \overline{S B V}_{y}^{p}$. By (3.1), there is a subsequence such that $\partial_{1} u_{k} \rightarrow D u \cdot e_{1}$ weakly in $L^{p}\left((0,1)^{2}\right)$, and in particular $D u \cdot e_{1} \in L^{p}\left((0,1)^{2}\right)$. Since $e_{1}$ is the normal to the Dirichlet boundary $\left\{x_{1}=0\right\}$, it follows as in the proof of Rellich's compact embedding theorem on the boundary that $u\left(0, x_{2}\right)=x_{2}$ in the sense of traces. Step 1 also yields $\partial_{2} u_{k} \stackrel{*}{\rightarrow} D u \cdot e_{2}$ in $\mathcal{M}((0,1) \times(\delta, 1-\delta))$. By (3.3), there are a subsequence and $v \in L^{p}\left((0,1)^{2}\right)$ such that $\left(\partial_{2} u_{k}\right) \chi_{A_{k}^{\delta}} \rightarrow v$ weakly in $L^{p}\left((0,1)^{2}\right)$. Hence, we have in $\mathcal{M}((0,1) \times(\delta, 1-\delta))$,

$0 \leq\left(\partial_{2} u_{k}\right) \chi_{B_{\delta}^{k}}=D u_{k} \cdot e_{2}-\left(\partial_{2} u_{k}\right) \chi_{A_{\delta}^{k}} \stackrel{*}{\rightarrow} D u \cdot e_{2}-v=D^{a c} u \cdot e_{2}+D^{S} u \cdot e_{2}-v$.

Since $D^{S} u \cdot e_{2} \perp\left(D^{a c} u \cdot e_{2}-v\right)$, we conclude that $D^{S} u \cdot e_{2} \geq 0$.

It remains to show that $u \in S B V_{\text {loc }}$ and that $D^{a c} u \cdot e_{2}-v \in L^{p}$. We refine the previous argument, and proceed by slicing (see [1, Theorems 3.107 and 3.108]), i.e., for almost every $x_{1}$, we consider the slice $u^{x_{1}}(\cdot):=u\left(x_{1}, \cdot\right)$ and show that it has locally a finite number of jumps, no Cantor part, and an absolutely continuous part which is contained in $L^{p}$.

By Fatou's lemma, the assumption $E_{\theta_{k}}\left(u_{k}\right) \leq M$ implies that there is $m \in$ $L^{1}((0,1))$ such that

$$
\liminf _{k \rightarrow \infty} \int_{0}^{1} \min \left\{\left|\left(u_{k}^{x_{1}}\right)^{\prime}\left(x_{2}\right)\right|^{p},\left|\left(u_{k}^{x_{1}}\right)^{\prime}\left(x_{2}\right)-\frac{1}{\theta_{k}}\right|^{p}\right\} \mathrm{d} x_{2}+\sigma \theta_{k}\left|\left(u_{k}^{x_{1}}\right)^{\prime \prime}\right|((0,1)) \leq m\left(x_{1}\right)
$$

for almost every $x_{1}$. Analogously, by (3.5) and (3.2), for any $\delta$ there is $m_{\delta} \in$ $L^{1}((0,1))$ such that

$$
\liminf _{k \rightarrow \infty}\left(\int_{\delta}^{1-\delta}\left|\left(u_{k}^{x_{1}}\right)^{\prime}\left(x_{2}\right)\right| \mathrm{d} x_{2}+\int_{0}^{1}\left|u_{k}^{x_{1}}\left(x_{2}\right)\right| \mathrm{d} x_{2}\right) \leq m_{\delta}\left(x_{1}\right)
$$

for almost every $x_{1}$. After extracting a further subsequence $u_{k}^{x_{1}} \rightarrow u^{x_{1}}$ in $L^{1}((0,1))$ for almost every $x_{1}$. Fix one such $x_{1} \in(0,1)$ and $\delta \in(0,1 / 2)$. By $(3.7)$ the sequence $u_{k}^{x_{1}}$ is bounded in $W^{1,1}((\delta, 1-\delta))$, therefore $u_{k}^{x_{1}} \stackrel{*}{\rightarrow} u^{x_{1}}$ in $B V((\delta, 1-\delta))$.

For $s \in \mathbb{R}$ we define

$$
P_{k}^{x_{1}}(s):=\left\{x_{2} \in(\delta, 1-\delta):\left(u_{k}^{x_{1}}\right)^{\prime}\left(x_{2}\right)>s\right\} .
$$

Condition (3.6) implies continuity of $u_{k}^{x_{1}}$, therefore for any $s \in \mathbb{R}$ the set $P_{k}^{x_{1}}(s)$ is a countable union of intervals. By the coarea formula one has

$$
\sigma \theta_{k} \int_{\mathbb{R}} \mathcal{H}^{0}\left(\partial_{(\delta, 1-\delta)} P_{k}^{x_{1}}(s)\right) \mathrm{d} s=\sigma \theta_{k}\left|\left(u_{k}^{x_{1}}\right)^{\prime \prime}\right|((\delta, 1-\delta)) .
$$


Here $\partial_{(\delta, 1-\delta)} P$ is the part of the measure-theoretic boundary of $P$ which is contained in $(\delta, 1-\delta)$. By (3.6), for large enough $k$ the quantity on the right is smaller than $2 m\left(x_{1}\right)$.

We fix $\eta \in(0,1 / 4)$, and choose $t_{k} \in\left[\eta / \theta_{k},(1-\eta) / \theta_{k}\right]$ which minimizes (in this interval) the quantity $\mathcal{H}^{0}\left(\partial_{(\delta, 1-\delta)} P_{k}^{x_{1}}(\cdot)\right)$. In particular, $P_{k}^{x_{1}}\left(t_{k}\right)$ is the union of at most $2 m\left(x_{1}\right) / \sigma$ disjoint open intervals. Let $\left\{y_{k}^{(j)}: j \in \mathcal{J}\right\}$ be the set of midpoints of the intervals that constitute $P_{k}^{x_{1}}\left(t_{k}\right)$. Since their number is bounded, after extracting a further subsequence we can assume $y_{k}^{(j)} \rightarrow z^{(j)}$, not all necessarily distinct. Let $K:=\left\{z^{(j)}: j \in \mathcal{J}\right\} \cap(\delta, 1-\delta)$. For later reference we note that

$$
2 \mathcal{H}^{0}(K) \leq \liminf _{k \rightarrow \infty} \mathcal{H}^{0}\left(\partial_{(\delta, 1-\delta)} P_{k}^{x_{1}}\left(t_{k}\right)\right) \leq \liminf _{k \rightarrow \infty} \frac{\theta_{k}}{1-2 \eta}\left|\left(u_{k}^{x_{1}}\right)^{\prime \prime}\right|((\delta, 1-\delta)) .
$$

Fix now $\varepsilon>0$, and let $I_{\varepsilon}=(\delta+\varepsilon, 1-\delta-\varepsilon) \backslash \bigcup_{j}\left(z^{(j)}-\varepsilon, z^{(j)}+\varepsilon\right)$. By (3.7) we have $\left|P_{k}^{x_{1}}\left(t_{k}\right)\right| \rightarrow 0$. Since $y_{k}^{(j)} \rightarrow z^{(j)}$, for $k$ large enough we have $P_{k}^{x_{1}}\left(t_{k}\right) \cap I_{\varepsilon}=\emptyset$. Since $\alpha \leq(1-\eta) / \theta_{k}$ implies $\alpha \leq \eta^{-1}\left|\alpha-1 / \theta_{k}\right|$ we obtain (for sufficiently large $k$ )

$$
\int_{I_{\varepsilon}}\left|\left(u_{k}^{x_{1}}\right)^{\prime}\right|^{p} \mathrm{~d} x_{2} \leq \frac{1}{\eta^{p}} \int_{0}^{1} \min \left\{\left|\left(u_{k}^{x_{1}}\right)^{\prime}\left(x_{2}\right)\right|^{p},\left|\left(u_{k}^{x_{1}}\right)^{\prime}\left(x_{2}\right)-\frac{1}{\theta_{k}}\right|^{p}\right\} \mathrm{d} x_{2} \leq \frac{2}{\eta^{p}} m\left(x_{1}\right) .
$$

Therefore $u_{k}^{x_{1}} \chi_{I_{\varepsilon}}$ has a weak limit in $W^{1, p}\left(I_{\varepsilon}\right)$, which coincides with $u^{x_{1}}$ and obeys $\left\|\left(u^{x_{1}}\right)^{\prime}\right\|_{L^{p}\left(I_{\varepsilon}\right)}^{p} \leq 2 \eta^{-p} m\left(x_{1}\right)$. Since $\varepsilon$ was arbitrary, we conclude that $u^{x_{1}} \in$ $W^{1, p}((\delta, 1-\delta) \backslash K)$; since the set of jump points is finite, this space is a subset of $S B V^{p}((\delta, 1-\delta)), u^{x_{1}}$ can only jump in the points of $K$, and the jumps are positive. Finally, since $\left\|\left(u^{x_{1}}\right)^{\prime}\right\|_{L^{p}((\delta, 1-\delta))}^{p} \leq 2 \eta^{-p} m\left(x_{1}\right)$ for all $\delta$, we conclude $\left\|\left(u^{x_{1}}\right)^{\prime}\right\|_{L^{p}((0,1))}^{p} \leq 2 \eta^{-p} m\left(x_{1}\right)$. Hence $u \in S B V_{\mathrm{loc}}^{p}\left((0,1)^{2}\right)$, with $\nabla u \in$ $L^{p}\left((0,1)^{2} ; \mathbb{R}^{2}\right)$.

Step 3: Lower bound. For the same slices $u^{x_{1}}$ as in Step 2, we have by (3.8)

$$
2 \sigma(1-2 \eta) \mathcal{H}^{0}\left((\delta, 1-\delta) \cap J_{u^{x_{1}}}\right) \leq \liminf _{k \rightarrow \infty} \sigma \theta_{k}\left|\left(u_{k}^{x_{1}}\right)^{\prime \prime}\right|((0,1)) .
$$

Since $\delta$ and $\eta \in(0,1 / 4)$ were arbitrary, it follows that $2 \sigma \mathcal{H}^{0}\left(J_{u^{x_{1}}}\right) \leq$ $\liminf _{k \rightarrow \infty} \sigma \theta_{k}\left|\left(u_{k}^{x_{1}}\right)^{\prime \prime}\right|((0,1))$. Recall from Step 2 that $D^{S} u \cdot e_{1}=0$, and thus we have

$$
\int_{0}^{1} \mathcal{H}^{0}\left(J_{u^{x_{1}}}\right) \mathrm{d} x_{1}=\mathcal{H}^{1}\left(J_{u}\right)
$$

Combining this with the weak $L^{p}$-convergences of the regular parts, we conclude 
by Fatou's lemma that

$$
\begin{aligned}
& E(u)=\int_{(0,1)^{2}}\left(\left|\partial_{1} u\right|^{p}+\left|\partial_{2} u\right|^{p}\right) \mathrm{d} x+2 \sigma \mathcal{H}^{1}\left(J_{u}\right) \\
\leq & \liminf _{k \rightarrow \infty} \int_{(0,1)^{2}}\left|\partial_{1} u_{k}\right|^{p} \mathrm{~d} x \\
& +\liminf _{k \rightarrow \infty} \int_{0}^{1}\left(\int_{0}^{1} \min \left\{\left|\left(u_{k}^{x_{1}}\right)^{\prime}\right|^{p},\left|\left(u_{k}^{x_{1}}\right)^{\prime}-\frac{1}{\theta_{k}}\right|^{p}\right\} \mathrm{d} x_{2}+\sigma \theta_{k}\left|\left(u_{k}^{x_{1}}\right)^{\prime \prime}\right|((0,1))\right) \mathrm{d} x_{1} \\
\leq & \liminf _{k \rightarrow \infty} E_{\theta_{k}}\left(u_{k}\right) .
\end{aligned}
$$

This finishes the proof.

Let us note that the problem does not admit global weak-* convergence in $B V\left((0,1)^{2}\right)$, as the following example shows. For details and discussions we refer to $[26]$.

Example 3.2. Let $\alpha \in(p /(p+1), 1)$, and set

$$
u_{k}\left(x_{1}, x_{2}\right):= \begin{cases}\frac{1}{\theta_{k}} x_{2}+\left(1-\frac{1}{\theta_{k}}\right)\left(1-\theta_{k}^{\alpha} x_{1}\right) & \text { if } x_{2} \geq 1-\theta_{k}^{\alpha} x_{1} \\ x_{2} & \text { otherwise. }\end{cases}
$$

Then $E_{\theta_{k}}\left(u_{k}\right) \leq C$ but $\left\|\partial_{2} u_{k}\right\|_{L^{1}\left((0,1)^{2}\right)}$ is unbounded, and hence there is no subsequence that converges weakly-* in $B V\left((0,1)^{2}\right)$.

This issue could be overcome by imposing periodic boundary conditions at top and bottom of the square, i.e., $u\left(x_{1}, 1\right)-u\left(x_{1}, 0\right)=1$.

Lemma 3.3. Let $u \in \overline{S B V}_{y}^{p}, \delta \in(0,1 / 2)$. Then $|D u|((0,1) \times(\delta, 1-\delta))<\infty$.

Proof. Let $\varphi \in C_{c}^{1}([0,1] \times(0,1) ;[0,1])$, with $\varphi=1$ on $[0,1] \times(\delta, 1-\delta)$. Then

$$
\int_{(0,1)^{2}} u \partial_{2} \varphi \mathrm{d} x=-\int_{(0,1)^{2}}\left(\partial_{2} u\right) \varphi \mathrm{d} x-\int_{(0,1)^{2} \cap J_{u}}[u] \varphi \mathrm{d} \mathcal{H}^{1} .
$$

Since $[u] \geq 0$ almost everywhere,

$$
\left|D^{J} u\right|((0,1) \times(\delta, 1-\delta)) \leq \int_{(0,1)^{2} \cap J_{u}}[u] \varphi \mathrm{d} \mathcal{H}^{1} \leq\left\|\partial_{2} u\right\|_{L^{1}\left((0,1)^{2}\right)}+\|\nabla \varphi\|_{L^{\infty}}\|u\|_{L^{1}\left((0,1)^{2}\right)} .
$$

\section{Upper bound}

In this section, we prove the upper bound, i.e., part (iii) of Theorem 1.2. We proceed in two steps, the main difficulty being the density result in Subsection 4.2. The lines of the proof are outlined in the proof of Theorem 4.1 below. 
Theorem 4.1. Let $\theta_{k} \rightarrow 0$ and $u \in \overline{S B V}_{y}^{p}$. Then there exist $u_{k} \in \mathcal{A}$ such that $u_{k} \rightarrow u$ in $L^{1}\left((0,1)^{2}\right)$ and $E(u) \geq \lim \sup _{k \rightarrow \infty} E_{\theta_{k}}\left(u_{k}\right)$.

In the proof we mainly work on the space $S B V_{y}^{p}(\Omega)$ defined in (2.1) and use on this space the functional

$$
E(u, \Omega):=\int_{\Omega}\left(\left|\partial_{1} u\right|^{p}+\left|\partial_{2} u\right|^{p}\right) \mathrm{d} x+2 \sigma \mathcal{H}^{1}\left(J_{u} \cap \Omega\right)
$$

which reduces to $E(u)$ if $\Omega=(0,1)^{2}$ and $u\left(0, x_{2}\right)=x_{2}$.

Proof. Let $u \in \overline{S B V}_{y}^{p}$, extended to $(-1,0) \times(0,1)$ by $u\left(x_{1}, x_{2}\right)=x_{2}$. Assume that $E(u)<\infty$, otherwise there is nothing to prove. For $\eta \in(0,1 / 10)$ we define

$$
u_{\eta}(x):=u\left(x_{1}-2 \eta, 2 \eta+(1-4 \eta) x_{2}\right)+2 \eta\left(2 x_{2}-1\right),
$$

so that $u_{\eta}(x)=x_{2}$ for $x_{1} \leq 2 \eta$, and let $\Omega:=(-\eta, 1+\eta) \times(-\eta, 1+\eta)$. With Lemma 3.3 one obtains $u \in S B V((-1,1) \times(-\eta, 1+\eta))$ and therefore $u_{\eta} \in S B V_{y}^{p}(\Omega)$. Further, $u_{\eta} \rightarrow u$ in $L^{1}\left((0,1)^{2}\right)$ and $E\left(u_{\eta}, \Omega\right) \rightarrow E(u)$ as $\eta \rightarrow 0$. By Theorem 4.11 below (on the set $\Omega$, with $R:=\eta$ and $U:=(-\eta, \eta) \times(0,1))$ there exists a sequence $v_{j} \in S B V_{y}^{p}(\Omega)$ with $v_{j} \rightarrow u_{\eta}$ in $L^{1}(\Omega), E\left(v_{j}, \Omega\right) \rightarrow E\left(u_{\eta}, \Omega\right)$, such that $J_{v_{j}}$ is locally a finite union of segments, and $v_{j}=u_{\eta}$ in $U$. In particular, $v_{j}\left(0, x_{2}\right)=x_{2}$. Since $(0,1)^{2} \subset \subset \Omega, J_{v_{j}} \cap(0,1)^{2}$ is a finite union of segments.

By Lemma 4.3 below, there exists a sequence $w_{\ell} \in S B V_{y}^{p}$ such that $w_{\ell} \rightarrow$ $v_{j}$ in $L^{1}\left((0,1)^{2}\right)$ as $\ell \rightarrow \infty, w_{\ell}$ is smooth away from its jump set with smooth traces on both sides of the jumps, and the jump set consists of finitely many segments. Further, $w_{\ell}\left(0, x_{2}\right)=v_{j}\left(0, x_{2}\right)$ and $E\left(w_{\ell}\right) \rightarrow E\left(v_{j}\right)$ as $\ell \rightarrow \infty$. For every $w_{\ell}$, by Proposition 4.2 below, there exists a recovery sequence $w_{\theta}^{\ell} \rightarrow w_{\ell}$ with $\lim \sup _{\theta \rightarrow 0} E_{\theta}\left(w_{\theta}^{\ell}\right) \leq E\left(w_{\ell}\right)$. Finally, taking a diagonal sequence, we obtain a recovery sequence for $u$.

\subsection{Functions whose jump sets consist of finitely many segments}

We first provide an explicit construction of a recovery sequence for a generic function $u \in S B V_{y}^{p}\left((0,1)^{2}\right)$ whose jump set consists of finitely many segments.

Proposition 4.2. Let $u \in S B V_{y}^{p}\left((0,1)^{2}\right)$ with $u\left(0, x_{2}\right)=x_{2}$ be such that $J_{u}$ is a finite union of segments, and $u$ smooth away from its jump set with smooth traces on both sides of the jumps. Then, for $\theta \in(0,1 / 2]$ there is $u_{\theta} \in \mathcal{A}$ such that $u_{\theta} \rightarrow u$ in $L^{1}\left((0,1)^{2}\right)$ as $\theta \rightarrow 0, \limsup _{\theta \rightarrow 0} E_{\theta}\left(u_{\theta}\right) \leq E(u)$ and $u_{\theta}\left(0, x_{2}\right)=x_{2}$ for all $\theta$.

Proof. Suppose that $J_{u}=\bigcup_{k=0}^{K}\left[a_{k}, b_{k}\right] \times\left\{y_{k}\right\}$ (up to a null set). Let $\rho>0$ be such that the distance between any pair of segments is larger than $2 \rho$. We shall modify $u$ inside each set $\left(a_{k}, b_{k}\right) \times\left(y_{k}-\rho, y_{k}+\rho\right)$, and not touch it outside. Set $h_{k}\left(x_{1}\right):=[u]\left(x_{1}, y_{k}\right) \geq 0$. We can assume without loss of generality that $\theta$ is so that $\theta\left\|h_{k}\right\|_{L^{\infty}} \leq \rho$ for all $k=0, \ldots, K$ (otherwise $u_{\theta}(x):=x_{2}$ will do). We set

$$
u_{\theta}(x):= \begin{cases}u(x)+\left(\frac{x_{2}-y_{k}}{\theta}-h_{k}\left(x_{1}\right)\right) & \text { if } y_{k}<x_{2}<y_{k}+\theta h_{k}\left(x_{1}\right), \\ u(x) & \text { otherwise. }\end{cases}
$$


If $u(0, \cdot)$ is continuous, then $u_{\theta}(0, \cdot)=u(0, \cdot)$. We set

$$
A_{\theta}:=\bigcup_{k=0}^{K} A_{\theta}^{k}, \quad A_{\theta}^{k}:=\left\{\left(x_{1}, x_{2}\right): x_{1} \in\left(a_{k}, b_{k}\right), y_{k}<x_{2}<\theta h_{k}\left(x_{1}\right)\right\}
$$

and observe that $\left|A_{\theta}\right| \leq \theta \sum_{k}\left\|h_{k}\right\|_{L^{\infty}} \rightarrow 0$ as $\theta \rightarrow 0$. Since $u=u_{\theta}$ outside $A_{\theta}$, we obtain

$$
\left\|u-u_{\theta}\right\|_{L^{1}\left((0,1)^{2}\right)} \leq\left|A_{\theta}\right| \max _{k}\left\|h_{k}\right\|_{L^{\infty}} \rightarrow 0 .
$$

It remains to prove convergence of the energies. Outside $A_{\theta}$ we have $\nabla u=\nabla u_{\theta}$. Inside $A_{\theta}$ we have $\left|\partial_{1} u_{\theta}\right| \leq\left\|\partial_{1} u\right\|_{L^{\infty}}+\max _{k}\left\|h_{k}^{\prime}\right\|_{L^{\infty}}$ and $\left|\partial_{2} u_{\theta}-\frac{1}{\theta}\right| \leq\left\|\partial_{2} u\right\|_{L^{\infty}}$, therefore

$$
\int_{(0,1)^{2}}\left|\partial_{1} u_{\theta}\right|^{p}+\min \left\{\left|\partial_{2} u_{\theta}\right|^{p},\left|\partial_{2} u_{\theta}-\frac{1}{\theta}\right|^{p}\right\} \mathrm{d} x \leq \int_{(0,1)^{2}}\left|\partial_{1} u\right|^{p}+\left|\partial_{2} u\right|^{p} \mathrm{~d} x+c\left|A_{\theta}\right| .
$$

We next consider the surface energy term. By construction, $D u_{\theta} \in S B V\left((0,1)^{2}\right)$ with $J_{D u_{\theta}} \subset \partial A_{\theta}$, so that $\mathcal{H}^{1}\left(J_{D u_{\theta}}\right) \leq \sum_{k} \int_{a_{k}}^{b_{k}}\left(1+\sqrt{1+\theta^{2}\left(h_{k}^{\prime}\right)^{2}(t)}\right) \mathrm{d} t \rightarrow 2 \sum_{k}\left(b_{k}-\right.$ $\left.a_{k}\right)=2 \mathcal{H}^{1}\left(J_{u}\right)$. At the same time, $\left|\left[D u_{\theta}\right]\right| \leq \theta^{-1}+\max _{k}\left\|h_{k}^{\prime}\right\|_{L^{\infty}}$. Further, $\nabla^{2} u_{\theta}=\nabla^{2} u+\nabla^{2} h_{k}\left(x_{1}\right) \chi_{A_{\theta}^{k}}$. Since $\left\|h_{k}^{\prime \prime}\right\|_{L^{\infty}} \leq c$ and $\left|A_{\theta}^{k}\right| \leq c \theta$, we estimate

$$
\begin{aligned}
\sigma \theta\left|D^{2} u_{\theta}\right|\left((0,1)^{2}\right) & \leq \sigma \theta\left(\left\|\nabla^{2} u\right\|_{L^{1}\left((0,1)^{2}\right)}+c\left|A_{\theta}\right|+\left(\frac{1}{\theta}+c\right) \mathcal{H}^{1}\left(J_{D u_{\theta}}\right)\right) \\
& \leq 2 \sigma(1+c \theta) \mathcal{H}^{1}\left(J_{u}\right)+c \sigma \theta
\end{aligned}
$$

Putting together (4.1) and (4.2), we obtain $\lim \sup _{\theta \rightarrow 0} E_{\theta}\left(u_{\theta}\right) \leq E(u)$. This concludes the proof.

Lemma 4.3. Let $u \in S B V_{y}^{p}\left((0,1)^{2}\right)$ be such that $J_{u}$ is a finite union of segments and such that $u\left(0, x_{2}\right)=x_{2}$. Then there is a sequence $v_{j} \in S B V_{y}^{p}\left((0,1)^{2}\right)$ with the following properties: We have $v_{j} \rightarrow u$ in $L^{1}$, each $v_{j}$ is smooth (up to the boundary) away from the jump set, which consists of finitely many segments, the traces on both sides of the jump are smooth and $v_{j}\left(0, x_{2}\right)=x_{2}$. Further, $\lim _{\sup _{j \rightarrow \infty}} E\left(v_{j}\right) \leq$ $E(u)$.

Proof. For $\varepsilon>0$ let $\varphi_{\varepsilon} \in C_{c}^{\infty}((-\varepsilon, \varepsilon))$ be a one-dimensional mollifier. Let $J_{u}=$ $\bigcup_{k=0}^{K}\left[a_{k}, b_{k}\right] \times\left\{y_{k}\right\}$ (up to null sets).

We first mollify in the horizontal direction. Extend $u$ by $u\left(x_{1}, x_{2}\right)=x_{2}$ for $x_{1} \leq 0$. We set, for $x \in(-\varepsilon, 1-\varepsilon) \times(0,1)$,

$$
w_{\varepsilon}\left(x_{1}, x_{2}\right):=\int_{\mathbb{R}} \varphi_{\varepsilon}\left(x_{1}-t\right) u\left(t, x_{2}\right) \mathrm{d} t .
$$

We remark that $w_{\varepsilon} \in S B V$ and its jump set is contained in the segments which arise from those where $u$ jumps by making them $\varepsilon$-longer on each side. Precisely, $J_{w_{\varepsilon}} \subset \bigcup_{k=0}^{K}\left[a_{k}-\varepsilon, b_{k}+\varepsilon\right] \times\left\{y_{k}\right\}$ (up to null sets), hence its length has grown 
by at most $2(K+1) \varepsilon$. By convexity $\left\|\partial_{i} w_{\varepsilon}\right\|_{L^{p}((-\varepsilon, 1-\varepsilon) \times(0,1))} \leq\left\|\partial_{i} u\right\|_{L^{p}\left((0,1)^{2}\right)}$ for $i=1,2$. Further, if $[u] \geq 0$ then $\left[w_{\varepsilon}\right] \geq 0$, since the new jump arises from the old one by averaging.

At this point we mollify in the vertical direction, separately in each region without discontinuities. In order to keep the boundary data it is convenient to subtract the affine function $x \mapsto x_{2}$. Let $a, b \in(0,1)$ be two consecutive elements of $\{0,1\} \cup\left\{y_{k}\right\}_{k=0, \ldots, K}$, i.e., two distinct values such that $(0,1) \times(a, b) \cap J_{u}$ is an $\mathcal{H}^{1}$-null set. We focus on the construction for $x_{2} \in(a, b)$, the other regions are analogous. We can assume $b>a+2 \varepsilon$. We set

$$
\hat{z}_{\varepsilon}\left(x_{1}, x_{2}\right):= \begin{cases}w_{\varepsilon}\left(x_{1}, a\right)-a & \text { if } x_{2} \leq a \\ w_{\varepsilon}\left(x_{1}, x_{2}\right)-x_{2} & \text { if } a<x_{2}<b \\ w_{\varepsilon}\left(x_{1}, b\right)-b & \text { if } x_{2} \geq b\end{cases}
$$

and $z_{\tilde{\varepsilon}}$ as the vertical mollification of $\hat{z}_{\varepsilon}$ on a scale $\tilde{\varepsilon} \leq \varepsilon$, i.e. $z_{\tilde{\varepsilon}}\left(x_{1}, x_{2}\right):=\int_{\mathbb{R}} \varphi_{\tilde{\varepsilon}}\left(x_{2}-\right.$ $t) \hat{z}_{\varepsilon}\left(x_{1}, t\right) \mathrm{d} t$. We remark that $z_{\tilde{\varepsilon}}\left(x_{1}, x_{2}\right)=w_{\varepsilon}\left(x_{1}, b\right)-b$ for all $x_{2} \geq b+\tilde{\varepsilon}$.

Finally, we scale back so that $(-\varepsilon, 1-\varepsilon) \times(a-\tilde{\varepsilon}, b+\tilde{\varepsilon})$ is mapped to $(0,1) \times(a, b)$. Precisely, we define

$$
\hat{v}_{\tilde{\varepsilon}}\left(x_{1}, x_{2}\right):=z_{\tilde{\varepsilon}}\left(x_{1}-\varepsilon, a-\tilde{\varepsilon}+\left(x_{2}-a\right) \frac{b+2 \tilde{\varepsilon}-a}{b-a}\right)+x_{2} .
$$

This gives a smooth map on the set $[0,1] \times[a, b]$, because the construction is the same as mollification (of an extension) with $\psi_{\varepsilon, \tilde{\varepsilon}}\left(x_{1}, x_{2}\right)=\varphi_{\varepsilon}\left(x_{1}\right) \varphi_{\tilde{\varepsilon}}\left(x_{2}\right) \in$ $C_{c}^{\infty}((-\varepsilon, \varepsilon) \times(-\tilde{\varepsilon}, \tilde{\varepsilon}))$, combined with an affine rescaling. The only term in the derivative that is not automatically estimated by convexity is the $x_{1}$-derivative close to the jumps. However, $\left\|\partial_{1} \hat{v}_{\tilde{\varepsilon}}\right\|_{L^{p}((0,1) \times(b-\tilde{\varepsilon}, b))}^{p} \leq\left\|\partial_{1} w_{\varepsilon}\right\|_{L^{p}((0,1) \times(b-\tilde{\varepsilon}, b))}^{p}+$ $\tilde{\varepsilon}\left\|\partial_{1} w_{\varepsilon}(b, \cdot)\right\|_{L^{p}((0,1))}^{p}$ where the second term gets small for small $\tilde{\varepsilon}$. The same is done in all other intervals. On the lines of the jumps, $\hat{v}_{\tilde{\varepsilon}}$ coincides with $w_{\varepsilon}$, which has a jump of the controlled length. Further, the jump has the appropriate sign and is smooth. Close to $a$ and $b$ the function $v_{\varepsilon}(x)-x_{2}$ does not depend on $x_{2}$, therefore the constructions on the two sides match smoothly away from the jump.

\subsection{Density}

In this section we prove density of functions with regular jump sets. The argument is more naturally discussed in general domains, but with a jump set of finite total length, therefore we use the space $S B V_{y}^{p}$ as introduced in (2.1). The density proof is done by three different constructions in small squares, that we present first, followed by a somewhat involved covering argument, discussed later. The constructions in turn build upon suitable variants of the Poincaré inequality.

We write $Q_{\rho}:=(-\rho, \rho)^{2}, Q_{\rho}(x):=x+Q_{\rho}$. For two functions $u, v: \Omega \rightarrow \mathbb{R}^{m}$ we say that $u=v$ around $\partial \Omega$ if there is $\omega \subset \subset \Omega$ such that $u=v$ in $\Omega \backslash \omega$. For the entire construction we fix a mollifier $\varphi_{\rho} \in C_{c}^{\infty}\left(Q_{\rho / 2} ;[0, \infty)\right)$ with $\left|D \varphi_{\rho}\right| \leq c / \rho^{3}$. 


\subsubsection{Constructions: Type I (bulk squares)}

We first treat squares which contain a small amount of jump set. Since the jump set is purely horizontal, one can use the normal Poincaré inequality to control the dependence of $u$ on $x_{1}$ (Lemma 4.4). At the same time, if the length of the jump set is not enough to divide the square into two halves, there are some vertical sections on which one can also use Poincaré. This gives a control of the $L^{p}$ oscillation of $u$ in terms of the $L^{p}$ norm of its regular gradient $\nabla u$ (Lemma 4.5), and therefore permits to estimate the gradient of a mollification (Lemma 4.6). An interpolation around the boundary leads to a construction which makes $u$ smooth in the interior of the square (Proposition 4.7), which is the main result of this subsection.

Lemma 4.4 (Horizontal Poincaré). If $u, v \in S B V_{y}^{p}\left(Q_{r}\left(x_{*}\right)\right)$ and $u=v$ on $\partial Q_{r}\left(x_{*}\right)$, then

$$
\|u-v\|_{L^{p}\left(Q_{r}\left(x_{*}\right)\right)} \leq c r\|\nabla u-\nabla v\|_{L^{p}\left(Q_{r}\left(x_{*}\right)\right)} .
$$

The same holds if $u=v$ on $\partial Q_{r}\left(x_{*}\right)$ is replaced by the fact that there is $t \in[-r, r]$ such that $u=v$ on $\left(x_{*}\right)+\{t\} \times(-r, r)$ in the sense of traces.

Proof. It suffices to apply the one-dimensional Poincaré estimate in the $x_{1}$-direction.

Lemma 4.5 (Poincaré with discontinuities). There is $c>0$ such that for any $\rho>0$ and any $u \in S B V_{y}^{p}\left(Q_{\rho}\left(x_{*}\right)\right)$ with $\mathcal{H}^{1}\left(J_{u} \cap Q_{\rho}\left(x_{*}\right)\right) \leq \rho$ there is $\bar{u} \in \mathbb{R}$ such that

$$
\|u-\bar{u}\|_{L^{p}\left(Q_{\rho}\left(x_{*}\right)\right)} \leq c \rho\|\nabla u\|_{L^{p}\left(Q_{\rho}\left(x_{*}\right)\right)} \cdot
$$

Proof. Without loss of generality $x_{*}=0$. Since horizontal slices of $u$ are in $W^{1, p}$, by the one-dimensional Poincaré inequality one has

$$
\int_{-\rho}^{\rho}\left|u\left(x_{1}, x_{2}\right)-u\left(x_{1}^{\prime}, x_{2}\right)\right|^{p} \mathrm{~d} x_{2} \leq(2 \rho)^{p-1} \int_{Q_{\rho}}\left|\partial_{1} u\right|^{p} \mathrm{~d} x
$$

for almost every $x_{1}, x_{1}^{\prime} \in(-\rho, \rho)$. For fixed $x_{1}$, consider now the vertical slices $t \mapsto u^{x_{1}}(t):=u\left(x_{1}, t\right)$. By the $S B V$ slicing theorem, since $\mathcal{H}^{1}\left(J_{u}\right) \leq \rho$, there is a set $E \subset(-\rho, \rho)$ of measure at least $\rho$ such that $u^{x_{1}} \in W^{1, p}((-\rho, \rho))$ for $x_{1} \in E$. Further, by Fubini's theorem there is a subset $E^{\prime} \subset E$ of measure at least $\rho / 2$ such that $\int_{-\rho}^{\rho}\left|\partial_{2} u\left(x_{1}^{\prime}, t\right)\right|^{p} \mathrm{~d} t \leq 2 / \rho \int_{Q_{\rho}}\left|\partial_{2} u\right|^{p} \mathrm{~d} x \quad$ for all $x_{1}^{\prime} \in E^{\prime}$. We choose $x_{1}^{\prime} \in E^{\prime}$ such that (4.5) holds for almost every $x_{1} \in(-\rho, \rho)$. By the one-dimensional Poincaré inequality there is $\bar{u} \in \mathbb{R}$ such that

$$
\int_{-\rho}^{\rho}\left|u\left(x_{1}^{\prime}, x_{2}\right)-\bar{u}\right|^{p} \mathrm{~d} x_{2} \leq(2 \rho)^{p} \int_{-\rho}^{\rho}\left|\partial_{2} u\left(x_{1}^{\prime}, x_{2}\right)\right|^{p} \mathrm{~d} x_{2} \leq 2^{p+1} \rho^{p-1} \int_{Q_{\rho}}\left|\partial_{2} u\right|^{p} \mathrm{~d} x .
$$

Combining with (4.5) and integrating in $x_{1}$ we conclude

$$
\int_{Q_{\rho}}\left|u\left(x_{1}, x_{2}\right)-\bar{u}\right|^{p} \mathrm{~d} x_{2} \leq c \rho^{p} \int_{Q_{\rho}}|\nabla u|^{p} \mathrm{~d} x .
$$

This finishes the proof. 
Lemma 4.6 (Mollification with small jump set). Let $u \in S B V_{y}^{p}\left(Q_{r}\left(x_{*}\right)\right)$, with $\mathcal{H}^{1}\left(J_{u} \cap Q_{r}\left(x_{*}\right)\right) \leq \rho$, for $0<\rho<r$. Then

$$
\int_{Q_{r-\rho}\left(x_{*}\right)}\left|\nabla\left(u * \varphi_{\rho}\right)\right|^{p} \mathrm{~d} x \leq\left(1+c \frac{\mathcal{H}^{1}\left(J_{u} \cap Q_{r}\left(x_{*}\right)\right)^{1 / p^{\prime}}}{\rho^{1 / p^{\prime}}}\right)^{p} \int_{Q_{r}\left(x_{*}\right)}|\nabla u|^{p} \mathrm{~d} x .
$$

Proof. We can assume $x_{*}=0$. Fix $x \in Q_{r-\rho}$. Since horizontal slices of $u$ are in $W^{1, p}$, we have

$$
\partial_{1}\left(u * \varphi_{\rho}\right)(x)=\int_{Q_{\rho}(x)} \partial_{1} u(y) \varphi_{\rho}(x-y) \mathrm{d} y=\left(\left(\partial_{1} u\right) * \varphi_{\rho}\right)(x) .
$$

The other component is more subtle, since $u$ jumps in the $x_{2}$ direction. Let $\omega_{x}:=\left\{y \in Q_{\rho}(x): y+\mathbb{R}_{2} \cap J_{u} \cap Q_{\rho}(x) \neq \emptyset\right\}$. We write

$$
\partial_{2}\left(u * \varphi_{\rho}\right)(x)=\int_{Q_{\rho}(x)} u(y) \partial_{2} \varphi_{\rho}(x-y) \mathrm{d} y
$$

and separate the integral into the part in $\omega_{x}$ and the part outside it. Outside $\omega_{x}$ we can integrate by parts in the $x_{2}$ direction. Therefore for any $\bar{u} \in \mathbb{R}$

$$
\partial_{2}\left(u * \varphi_{\rho}\right)(x)=\int_{Q_{\rho}(x) \backslash \omega_{x}} \partial_{2} u(y) \varphi_{\rho}(x-y) \mathrm{d} y+\int_{\omega_{x}} \partial_{2} \varphi_{\rho}(x-y)(u(y)-\bar{u}) \mathrm{d} y .
$$

Let $R(x):=\int_{\omega_{x}} \partial_{2} \varphi_{\rho}(x-y)(u(y)-\bar{u}) \mathrm{d} y$ denote the last term. Combining with (4.6) gives

$\nabla\left(u * \varphi_{\rho}\right)(x)=\int_{Q_{\rho}(x) \backslash \omega_{x}} \nabla u(y) \varphi_{\rho}(x-y) \mathrm{d} y+\int_{\omega_{x}}\left(\partial_{1} u, 0\right)(y) \varphi_{\rho}(x-y) \mathrm{d} y+R(x) e_{2}$ which immediately gives

$$
\left|\nabla\left(u * \varphi_{\rho}\right)\right|(x) \leq\left(|\nabla u| * \varphi_{\rho}\right)(x)+|R|(x) .
$$

It remains to estimate $R$. Since $\left|\nabla \varphi_{\rho}\right| \leq c / \rho^{3}$, Hölder's inequality yields

$$
|R|(x) \leq \frac{c}{\rho^{3}}\|u-\bar{u}\|_{L^{p}\left(Q_{\rho}(x)\right)}\left|\omega_{x}\right|^{1 / p^{\prime}}
$$

where as usual $p^{\prime}=p /(p-1)$. By Lemma 4.5, with the appropriate choice of $\bar{u}$ we have $\|u-\bar{u}\|_{L^{p}\left(Q_{\rho}(x)\right)} \leq c \rho\|\nabla u\|_{L^{p}\left(Q_{\rho}(x)\right)}$. We observe that $\left|\omega_{x}\right| \leq 2 \rho \mathcal{H}^{1}\left(J_{u} \cap Q_{\rho}(x)\right)$ and $\|\nabla u\|_{L^{p}\left(Q_{\rho}(x)\right)}^{p}=\left(\chi_{Q_{\rho}} *|\nabla u|^{p}\right)(x)$, where $Q_{\rho}=Q_{\rho}(0)$. Therefore

$$
|R|^{p}(x) \leq \frac{c \mathcal{H}^{1}\left(J_{u} \cap Q_{r}\right)^{p / p^{\prime}}}{\rho^{2 p-p / p^{\prime}}}\left(\chi_{Q_{\rho}} *|\nabla u|^{p}\right)(x)
$$

for all $x \in Q_{r-\rho}$. Integrating over $x$ leads to

$$
\|R\|_{L^{p}\left(Q_{r-\rho}\right)}^{p} \leq \frac{c \mathcal{H}^{1}\left(J_{u} \cap Q_{r}\right)^{p / p^{\prime}}}{\rho^{2 p-p / p^{\prime}}} \rho^{2}\|\nabla u\|_{L^{p}\left(Q_{r}\right)}^{p}
$$


and, recalling (4.7) and using $2-1 / p^{\prime}-2 / p=1 / p^{\prime}$, we conclude

$$
\left\|\nabla\left(u * \varphi_{\rho}\right)\right\|_{L^{p}\left(Q_{r-\rho}\right)} \leq\|\nabla u\|_{L^{p}\left(Q_{r}\right)}+\frac{c \mathcal{H}^{1}\left(J_{u} \cap Q_{r}\right)^{1 / p^{\prime}}}{\rho^{1 / p^{\prime}}}\|\nabla u\|_{L^{p}\left(Q_{r}\right)} .
$$

We are now in the position to state the main result of this subsection.

Proposition 4.7. Let $u \in S B V_{y}^{p}\left(Q_{r}\right)$ with $\mathcal{H}^{1}\left(J_{u} \cap Q_{r}\right) \leq \rho \leq r / 10$. Then there is $v \in S B V_{y}^{p}\left(Q_{r}\right)$ such that $v \in W^{1, p}\left(Q_{r / 2}\right), v=u$ around $\partial Q_{r}, \mathcal{H}^{1}\left(J_{v} \backslash J_{u}\right)=0$,

$$
\|\nabla v\|_{L^{p}\left(Q_{r}\right)} \leq\left(1+c\left(\frac{\mathcal{H}^{1}\left(J_{u} \cap Q_{r}\right)}{\rho}\right)^{1 / p^{\prime}}+c \frac{\rho}{r}\right)\|\nabla u\|_{L^{p}\left(Q_{r}\right)},
$$

and $|D v|\left(Q_{r}\right) \leq c|D u|\left(Q_{r}\right)$.

Proof. We select $R \in(r / 2, r-3 \rho)$ such that

$$
\|\nabla u\|_{L^{p}\left(Q_{R+3 \rho} \backslash Q_{R-2 \rho}\right)}^{p} \leq \frac{c \rho}{r}\|\nabla u\|_{L^{p}\left(Q_{r}\right)}^{p} .
$$

Fix $\psi \in C_{c}^{\infty}\left(Q_{R+\rho} ;[0,1]\right)$ with $\psi=1$ in $Q_{R}$ and $|\nabla \psi| \leq c / \rho$. We set

$$
v:=\psi\left(u * \varphi_{\rho}\right)+(1-\psi) u,
$$

so that $J_{v} \subset J_{u} \backslash Q_{R}$ and $[v]=(1-\psi)[u]$ on $J_{v}$ (both up to $\mathcal{H}^{1}$-null sets), which implies $v \in S B V_{y}^{p}\left(Q_{r}\right)$. Inside $Q_{R}$ we have $v=u * \varphi_{\rho} \in C^{\infty}$, outside $Q_{R+\rho}$ we have $v=u$. By Lemma 4.6 applied to $Q_{R+\rho}$ we have $\|\nabla v\|_{L^{p}\left(Q_{R}\right)} \leq$ $\|\nabla u\|_{L^{p}\left(Q_{R+\rho}\right)}\left(1+c\left(\mathcal{H}^{1}\left(J_{u}\right) / \rho\right)^{1 / p^{\prime}}\right)$.

The elastic energy in the interpolation region is controlled by

$$
\begin{aligned}
\|\nabla v\|_{L^{p}\left(Q_{R+\rho} \backslash Q_{R}\right)}^{p} \leq & c\left\|\nabla\left(u * \varphi_{\rho}\right)\right\|_{L^{p}\left(Q_{R+\rho} \backslash Q_{R}\right)}^{p}+c\|\nabla u\|_{L^{p}\left(Q_{R+\rho} \backslash Q_{R}\right)}^{p} \\
& +\frac{c}{\rho^{p}}\left\|\left(u * \varphi_{\rho}\right)-u\right\|_{L^{p}\left(Q_{R+\rho} \backslash Q_{R}\right)}^{p} .
\end{aligned}
$$

We can cover this set by $c\lfloor r / \rho\rfloor$ squares $Q_{\rho}\left(y_{i}\right)$ of side length $2 \rho$, such that the larger squares $Q_{2 \rho}\left(y_{i}\right)$ have finite overlap and are contained in $Q_{R+3 \rho} \backslash Q_{R-2 \rho}$. By Lemma 4.6 with $r=2 \rho$ we have, for each $i,\left\|\nabla\left(u * \varphi_{\rho}\right)\right\|_{L^{p}\left(Q_{\rho}\left(y_{i}\right)\right)} \leq c\|\nabla u\|_{L^{p}\left(Q_{2 \rho}\left(y_{i}\right)\right)}$. By Lemma 4.5 applied to $Q_{2 \rho}\left(y_{i}\right)$ there is $\bar{u} \in \mathbb{R}$ with $\|u-\bar{u}\|_{L^{p}\left(Q_{2 \rho}\left(y_{i}\right)\right)} \leq$ $c \rho\|\nabla u\|_{L^{p}\left(Q_{2 \rho}\left(y_{i}\right)\right)}$. This implies $\left\|\varphi_{\rho} * u-\bar{u}\right\|_{L^{p}\left(Q_{\rho}\left(y_{i}\right)\right)} \leq c \rho\|\nabla u\|_{L^{p}\left(Q_{2 \rho}\left(y_{i}\right)\right)}$, and therefore $\left\|\varphi_{\rho} * u-u\right\|_{L^{p}\left(Q_{\rho}\left(y_{i}\right)\right)} \leq c \rho\|\nabla u\|_{L^{p}\left(Q_{2 \rho}\left(y_{i}\right)\right)}$. Summing over all squares we obtain

$$
\|\nabla v\|_{L^{p}\left(Q_{R+\rho} \backslash Q_{R}\right)}^{p} \leq c\|\nabla u\|_{L^{p}\left(Q_{R+3 \rho} \backslash Q_{R-2 \rho}\right)}^{p} \leq \frac{c \rho}{r}\|\nabla u\|_{L^{p}\left(Q_{r}\right)}^{p} .
$$

Collecting terms, we have

$\|\nabla v\|_{L^{p}\left(Q_{r}\right)}^{p} \leq\left(1+c\left(\mathcal{H}^{1}\left(J_{u}\right) / \rho\right)^{1 / p^{\prime}}\right)^{p}\|\nabla u\|_{L^{p}\left(Q_{R+\rho}\right)}^{p}+\frac{c \rho}{r}\|\nabla u\|_{L^{p}\left(Q_{r}\right)}^{p}+\|\nabla u\|_{L^{p}\left(Q_{r} \backslash Q_{R+\rho}\right)}^{p}$ 
which concludes the proof of the first estimate. To control the total variation of $D v$, by convexity one only needs to estimate the term $\left\|\left(u-u * \varphi_{\rho}\right) D \psi\right\|_{L^{1}\left(Q_{r}\right)}$, which is done using the Poincaré-type estimate $\left\|u-u * \varphi_{\rho}\right\|_{L^{1}\left(Q_{r-\rho}\right)} \leq c \rho|D u|\left(Q_{r}\right)$ and $|D \psi| \leq c / \rho$.

\subsubsection{Constructions: Type II (bad squares)}

We consider here squares where a substantial amount of jump set is present, without making any other structural assumption. The part where the jump set is approximately flat will be treated later, so that this construction will be used only on "bad" squares, where the jump set is present but irregular (on the scale of the square).

Proposition 4.8. For any $u \in S B V_{y}^{p}\left(Q_{r}\right)$ there is $v \in S B V_{y}^{p}\left(Q_{r}\right)$ with $u=$ $v$ around $\partial Q_{r},\|\nabla v\|_{L^{p}\left(Q_{r}\right)} \leq c\|\nabla u\|_{L^{p}\left(Q_{r}\right)}, \mathcal{H}^{1}\left(J_{v}\right) \leq c \mathcal{H}^{1}\left(J_{u}\right),|D v|\left(Q_{r}\right) \leq$ $c|D u|\left(Q_{r}\right)$, and such that $J_{v} \subset S \cup\left(J_{u} \backslash Q_{r / 2}\right) \cup N$, with $S$ the union of at most $c \mathcal{H}^{1}\left(J_{u} \cap Q_{r}\right) / r$ horizontal segments and $\mathcal{H}^{1}(N)=0$.

Proof. Pick $s \in(-r, r)$ such that $[u]\left(s, x_{2}\right) \geq 0$ for all $x_{2}$,

$$
\begin{aligned}
\sum_{J_{u} \cap\left(s e_{1}+\mathbb{R} e_{2}\right)}[u] \leq \frac{c}{r} \int_{J_{u}}[u] \mathrm{d} \mathcal{H}^{1}, & \mathcal{H}^{0}\left(J_{u} \cap\left(s e_{1}+\mathbb{R} e_{2}\right)\right) & \leq \frac{c}{r} \mathcal{H}^{1}\left(J_{u} \cap Q_{r}\right), \\
\text { and } & \int_{-r}^{r}\left|\partial_{2} u\right|^{p}\left(s, x_{2}\right) \mathrm{d} x_{2} & \leq \frac{c}{r} \int_{Q_{r}}\left|\partial_{2} u\right|^{p} \mathrm{~d} x,
\end{aligned}
$$

in the sense of slicing. Set $w\left(x_{1}, x_{2}\right):=u\left(s, x_{2}\right)$. Then $w \in S B V^{p}\left(Q_{r}\right), S:=J_{w}$ is the union of at most $\frac{c}{r} \mathcal{H}^{1}\left(J_{u}\right)$ horizontal segments of length $2 r$ and $\|\nabla w\|_{L^{p}\left(Q_{r}\right)} \leq$ $c\|\nabla u\|_{L^{p}\left(Q_{r}\right)}$. Moreover,

$$
\left|D^{J} w\right|\left(Q_{r}\right)=\int_{J_{w}}[w] \mathrm{d} \mathcal{H}^{1}=2 r \sum_{J_{u} \cap\left(s e_{1}+\mathbb{R} e_{2}\right)}[u] \leq c \int_{J_{u}}[u] \mathrm{d} \mathcal{H}^{1} .
$$

It remains to interpolate. We fix $\psi \in C_{c}^{\infty}\left(Q_{r} ;[0,1]\right)$ with $\psi=1$ on $Q_{r / 2}$ and $|\nabla \psi| \leq c / r$, and set

$$
v:=\psi w+(1-\psi) u,
$$

so that $J_{v} \subset\left(J_{u} \backslash Q_{r / 2}\right) \cup J_{w} \cup N$ with $\mathcal{H}^{1}(N)=0$. Further, from $[v]=\psi[w]+$ $(1-\psi)[u]$ and $0 \leq \psi \leq 1$ one easily verifies that $[u] \geq 0$ implies $[v] \geq 0$, hence $v \in S B V_{y}^{p}\left(Q_{r}\right)$ with $\mathcal{H}^{1}\left(J_{v}\right) \leq c \mathcal{H}^{1}\left(J_{u}\right)$.

By the Poincaré inequality in the $x_{1}$ direction (Lemma 4.4), $w=u$ on $\left\{x_{1}=s\right\}$ and the choice of $s$, we have

$$
\|w-u\|_{L^{p}\left(Q_{r}\right)} \leq c r\|\nabla u\|_{L^{p}\left(Q_{r}\right)} .
$$

Therefore

$$
\|\nabla v\|_{L^{p}\left(Q_{r}\right)} \leq c\|\nabla u\|_{L^{p}\left(Q_{r}\right)}+\frac{c}{r}\|w-u\|_{L^{p}\left(Q_{r}\right)}+\|\nabla w\|_{L^{p}\left(Q_{r}\right)} \leq c\|\nabla u\|_{L^{p}\left(Q_{r}\right)} .
$$

The estimate for $|D v|$ is done as in Proposition 4.7, using (4.8). 


\subsubsection{Constructions: Type III (jump squares)}

We finally deal with squares that contain regular parts of $J_{u}$, in the sense of blowups. Although the normal is almost everywhere $e_{2}$, the main part of the jump set needs not be a segment, even locally, but can be a countable union of segments contained in a $C^{1}$ curve.

Proposition 4.9. Let $u \in S B V_{y}^{p}\left(Q_{r}\right)$ and $\rho \in(0, r / 4)$ be such that there is $\gamma \in C^{1}((-r, r) ;(-r / 2, r / 2))$ with $\mathcal{H}^{1}\left(J_{u} \Delta\left\{\left(x_{1}, \gamma\left(x_{1}\right)\right): x_{1} \in(-r, r)\right\}\right)<\rho / 4$.

Then there is $v \in S B V_{y}^{p}\left(Q_{r}\right)$ such that $u=v$ around $\partial Q_{r}, J_{v} \subset\left(J_{u} \backslash\right.$ $\left.Q_{r-\rho}\right) \cup S \cup N$, with $S$ the union of two segments and $\mathcal{H}^{1}(N)=0,\|\nabla v\|_{L^{p}\left(Q_{r}\right)} \leq$ $c(r / \rho)\|\nabla u\|_{L^{p}\left(Q_{r}\right)}, \mathcal{H}^{1}\left(J_{v} \cap Q_{r-\rho}\right) \leq 2 r+2 \rho$, and $\mathcal{H}^{1}\left(J_{v} \backslash Q_{r-\rho}\right) \leq c \rho$.

Proof. For $x_{+} \in(r-\rho, r)$ we consider the slice $u^{x_{+}}(\cdot):=u\left(x_{+}, \cdot\right)$. By assumption, the set of $x_{+}$such that the jump set of $u^{x_{+}}$does not coincide with $\left\{\gamma\left(x_{+}\right)\right\}$has measure no larger than $\rho / 4$. By Fubini, the set of $x_{+}$such that $\left\|\left(u^{x_{+}}\right)^{\prime}\right\|_{L^{p}((-r, r))}^{p} \geq$ $\frac{2}{\rho}\|\nabla u\|_{L^{p}\left(Q_{r}\right)}^{p}$ has measure no larger than $\rho / 2$. Further, for almost all $x_{+},\left[u^{x_{+}}\right] \geq 0$ on its jump set. The same holds on the other side. Therefore we can choose $x_{-} \in(-r,-r+\rho)$ and $x_{+} \in(r-\rho, r)$ such that

$$
J_{u^{x_{ \pm}}}=\left\{y_{ \pm}\right\}, \quad\left[u^{x_{ \pm}}\right]\left(y_{ \pm}\right) \geq 0, \quad \text { and } \quad\left\|\left(u^{x_{ \pm}}\right)^{\prime}\right\|_{L^{p}((-r, r))}^{p} \leq \frac{2}{\rho}\|\nabla u\|_{L^{p}\left(Q_{r}\right)}^{p},
$$

where $y_{ \pm}:=\gamma\left(x_{ \pm}\right) \in(-r / 2, r / 2)$.

Let $\delta \in(0, r / 2)$, chosen below. We define $w: Q_{r} \rightarrow \mathbb{R}$ as $u$ outside $\left(x_{-}, x_{+}\right) \times$ $(-r, r)$, as the value at $x_{1}=x_{ \pm}$in $\left(\left(x_{-}, x_{+}\right) \backslash(-\delta, \delta)\right) \times(-r, r)$, and as the linear interpolation inside. Precisely,

$$
w\left(x_{1}, x_{2}\right):= \begin{cases}u\left(x_{1}, x_{2}\right) & \text { if }-r \leq x_{1}<x_{-} \\ u\left(x_{-}, x_{2}\right) & \text { if } x_{-} \leq x_{1} \leq-\delta \\ \frac{x_{1}+\delta}{2 \delta} u\left(x_{+}, x_{2}\right)+\frac{\delta-x_{1}}{2 \delta} u\left(x_{-}, x_{2}\right) & \text { if }-\delta<x_{1}<\delta \\ u\left(x_{+}, x_{2}\right) & \text { if } \delta \leq x_{1} \leq x_{+} \\ u\left(x_{1}, x_{2}\right) & \text { if } x_{+} \leq x_{1}<r .\end{cases}
$$

We observe that $J_{w} \cap\left(\left(x_{-}, x_{+}\right) \times(-r, r)\right)$ is the union of two segments of length $x_{+}+\delta$ and $\delta-x_{-}$, located at $x_{2}=y_{ \pm}$. Further, the jump of $w$ is a convex combination of the jumps of $u^{x_{ \pm}}$, therefore nonnegative, so that $w \in S B V_{y}^{p}\left(Q_{r}\right)$. We now estimate the derivatives. By convexity, $\left|\partial_{2} w\right|\left(x_{1}, x_{2}\right) \leq\left|\partial_{2} u\right|\left(x_{-}, x_{2}\right)+$ $\left|\partial_{2} u\right|\left(x_{+}, x_{2}\right)$ for all $x_{1} \in\left(x_{-}, x_{+}\right)$. The horizontal derivative vanishes outside $(-\delta, \delta)$, and obeys

$$
\left|\partial_{1} w\right|\left(x_{1}, x_{2}\right)=\frac{\left|u\left(x_{+}, x_{2}\right)-u\left(x_{-}, x_{2}\right)\right|}{2 \delta} \leq \frac{1}{2 \delta} \int_{x_{-}}^{x_{+}}\left|\partial_{1} u\right|\left(x_{1}, x_{2}\right) \mathrm{d} x_{1}
$$

inside, which implies, using first Hölder's inequality and then integrating,

$$
\|\nabla w\|_{L^{p}\left(\left(x_{-}, x_{+}\right) \times(-r, r)\right)}^{p} \leq c\left(\left(\frac{r}{\delta}\right)^{p}+\frac{r}{\rho}\right)\|\nabla u\|_{L^{p}\left(Q_{r}\right)}^{p} .
$$


Further, by Lemma 4.4

$$
\|u-w\|_{L^{p}\left(Q_{r}\right)} \leq c r\|\nabla u-\nabla w\|_{L^{p}\left(Q_{r}\right)} .
$$

Let now $\psi \in C_{c}^{\infty}((-r, r))$ with $\psi=1$ on $(-r+\rho, r-\rho)$ and $\left|\psi^{\prime}\right| \leq c / \rho$, and define

$$
v(x):=\psi\left(x_{2}\right) w(x)+\left(1-\psi\left(x_{2}\right)\right) u(x) .
$$

Obviously $v \in S B V_{y}^{p}\left(Q_{r}\right)$, and the jump has the stated properties. In particular, $\mathcal{H}^{1}\left(J_{v} \backslash Q_{r-\rho}\right) \leq 3 \rho$ because $\gamma^{\prime}=0 \mathcal{H}^{1}$-almost everywhere on the set $\left.\left\{x_{1}:\left(x_{1}, \gamma\left(x_{1}\right)\right) \in J_{u}\right)\right\}$, and $\mathcal{H}^{1}\left(J_{u} \Delta\left\{\left(x_{1}, \gamma\left(x_{1}\right)\right): x_{1} \in(-r, r)\right\}\right)<\rho / 4$. The energy of the interpolation is controlled by

$$
\|\nabla v\|_{L^{p}\left(Q_{r}\right)} \leq c\left(\frac{r}{\rho}+\frac{r}{\delta}+\left(\frac{r}{\rho}\right)^{1 / p}\right)\|\nabla u\|_{L^{p}\left(Q_{r}\right)} .
$$

We finally choose $\delta:=\rho$ and conclude the proof.

\subsubsection{Covering and global approximation}

We start with a covering Lemma. We need to cover an open set $\Omega$ by a family of squares which have finite overlap, such that the half-as-large squares still cover $\Omega$, and such that the "overlap chains" are bounded. For this purpose we define the set of $k$-neighbouring squares $\mathcal{N}_{k}(q)$ and state some of its properties. For a square $q \subset \mathbb{R}^{2}$ we denote by $\ell_{q}$ its half side length, so that $q=Q_{\ell_{q}}(x)$ for some $x$.

Lemma 4.10 (Covering). Let $\Omega \subset \mathbb{R}^{2}$ open, $\delta>0$. Then there are $N$ families of squares $\mathcal{F}_{1}, \ldots, \mathcal{F}_{N}$, all contained in $\Omega$ and with side length no larger than $\delta$, such that, with $\mathcal{F}:=\cup_{k} \mathcal{F}_{k}$ and $\hat{q}$ denoting the square with the same center and half the side length as $q$,

(i) $\Omega=\bigcup_{q \in \mathcal{F}} \hat{q}$;

(ii) if $q \cap q^{\prime} \neq \emptyset$, then $\frac{1}{c} \ell_{q} \leq \ell_{q^{\prime}} \leq c \ell_{q}$, for all $q, q^{\prime} \in \mathcal{F}$;

(iii) for each $k$ the squares in $\mathcal{F}_{k}$ are disjoint.

Further, for $q \in \mathcal{F}$ let $\mathcal{N}_{1}(q):=\left\{q^{\prime} \in \mathcal{F}: q \cap q^{\prime} \neq \emptyset\right\}$ be the set of its first neighbours, and $\mathcal{N}_{k+1}(q):=\cup_{q^{\prime} \in \mathcal{N}_{k}(q)} \mathcal{N}_{1}\left(q^{\prime}\right)$ be the set of k-neighbours. Then $\# \mathcal{N}_{k}(q) \leq b^{k}$ and $\operatorname{dist}\left(q, q^{\prime}\right)+\ell_{q^{\prime}} \leq a^{k} \ell_{q}$ for all $q^{\prime} \in \mathcal{N}_{k}(q)$.

The constants $N, a, b$ and $c$ are universal.

Proof. This is a variant of Whitney's covering argument, similar to the one used for example in [28, Theorem 3.1]. For a proof we refer to the variant of Whitney's covering presented in [41, pp. $167 \mathrm{ff}$.$] . In the definition of \Omega_{k}$ set $c=3$, and take the squares obtained there (possibly uniformly subdivided into smaller squares to ensure the maximal radius $\delta$ ) as $\hat{q}$. This easily gives the first and the second assertion. 
To estimate the number of neighbours fix a square $q$ and let $\left\{q_{i}\right\}$ be those squares that intersect $q$. Each square $q_{i}$ is contained in the square with the same center as $q$ and radius $2 c \ell_{q}$ and so are the disjoint smaller squares $\hat{q}_{i}$. Since additionally $\ell_{\hat{q}_{i}} \geq \ell_{q} /(2 c)$ we can conclude that their number is uniformly bounded. Property (iii) follows immediately, the bound on the distance of neighbours follows from (ii).

Theorem 4.11 (Jump set made of segments). Let $u \in S B V_{y}^{p}(\Omega)$, where $\Omega \subset \mathbb{R}^{2}$ is a Lipschitz bounded set. Then there is a sequence $v_{j} \in S B V_{y}^{p}(\Omega)$ with $v_{j} \rightarrow u$ in $L^{1}, u=v_{j}$ on $\partial \Omega, \limsup _{j} E\left(v_{j}, \Omega\right) \leq E(u, \Omega)$, such that $J_{v_{j}}$ is locally a finite union of segments.

Moreover: If $U \subset \Omega$ open is such that $B_{R}(U) \cap J_{u} \cap \Omega=\emptyset$ for some $R>0$, then the sequence $v_{j}$ can be constructed in a way that $v_{j}=u$ in $U$.

We say that $J_{v}$ is locally a finite union of segements, if for any $\omega \subset \subset \Omega$ there is a finite union of segments $S \subset \mathbb{R}^{2}$ such that $J_{v} \cap \omega$ coincides, up to $\mathcal{H}^{1}$-null sets, with $S \cap \omega$.

Proof. Step 1: Treatment of the main part of the jump set.

Since $J_{u}$ is rectifiable there are countably many curves $\gamma_{j} \in C^{1}(\mathbb{R})$ such that $J_{u} \subset \cup_{i}\left\{\left(x_{1}, \gamma_{i}\left(x_{1}\right): x_{1} \in \mathbb{R}\right\} \cup N\right.$, with $\mathcal{H}^{1}(N)=0$. All curves can be taken as graphs with respect to $x_{1}$, since for almost every $x \in J_{u}$ the normal is $e_{2}$.

Fix $\varepsilon \in(0,1 / 4)$. For $\mathcal{H}^{1}$-almost every $x \in J_{u}$ there is a curve $\gamma_{x} \in C^{1}(\mathbb{R})$ with $\gamma_{x}^{\prime}\left(x_{1}\right)=0$ such that

$$
\lim _{r \rightarrow 0} \frac{1}{2 r} \mathcal{H}^{1}\left(\left(J_{u} \Delta \gamma_{x}^{*}\right) \cap Q_{r}(x)\right)=0
$$

and

$$
\lim _{r \rightarrow 0} \frac{1}{2 r}\|\nabla u\|_{L^{p}\left(Q_{r}(x)\right)}^{p}=0 .
$$

Here we write $\gamma_{x}^{*}$ for the graph $\left\{\left(s, \gamma_{x}(s)\right): s \in \mathbb{R}\right\}$ of the curve $\gamma_{x}$. Since for almost every $r>0$ one has $\mathcal{H}^{1}\left(J_{u} \cap \partial Q_{r}(x)\right)=0$, there is a fine cover of $\mathcal{H}^{1}$-almost all of $J_{u}$ with squares $q$ such that

$$
\mathcal{H}^{1}\left(\left(J_{u} \Delta \gamma_{q}^{*}\right) \cap q\right) \leq \frac{\varepsilon \ell_{q}}{4}, \mathcal{H}^{1}\left(J_{u} \cap \partial q\right)=0, \gamma_{q}^{*} \cap q \subset R_{q}^{\varepsilon} \text {, and }\|\nabla u\|_{L^{p}(q)}^{p} \leq \varepsilon^{p+1} \ell_{q} .
$$

Here we denote by $\gamma_{q}$ the curve pertaining to the midpoint of $q$, and by $R_{q}^{\varepsilon}:=$ $x+\left(-\ell_{q}, \ell_{q}\right) \times\left(-\varepsilon \ell_{q}, \varepsilon \ell_{q}\right)$ the central stripe of $q$. The first condition implies $\mathcal{H}^{1}\left(J_{u} \cap q\right) \geq(1-\varepsilon / 4) \ell_{q}$. We can extract a countable set of disjoint squares which cover $\mathcal{H}^{1}$-almost all of $J_{u}$, and in particular a finite set $\mathcal{G}:=\left\{q_{1}, \ldots, q_{M}\right\}$ of disjoint squares with the property (4.9) which cover $J_{u}$ up to a set of measure $\varepsilon$.

We apply Proposition 4.9 to each square $q \in \mathcal{G}$, with $\rho:=\varepsilon \ell_{q}$, and define $w$ as the result in each $q$, and as $u$ outside. We obtain $w \in S B V_{y}^{p}(\Omega)$ which coincides with $u$ around $\partial \Omega$, and such that for any $q \in \mathcal{G}$ the set $J_{w} \cap \hat{q}^{(1-\varepsilon)}$ is the union of 
two segments. Here $\hat{q}^{(1-\varepsilon)}$ is the square with the same center as $q$ and side length $1-\varepsilon$ times smaller. The jump set is estimated by

$$
\begin{aligned}
\mathcal{H}^{1}\left(J_{w} \backslash \cup_{q \in \mathcal{G}} \hat{q}^{(1-\varepsilon)}\right) & \leq \mathcal{H}^{1}\left(J_{w} \backslash \cup_{q \in \mathcal{G}} q\right)+\sum_{q \in \mathcal{G}} \mathcal{H}^{1}\left(J_{w} \cap q \backslash \hat{q}^{(1-\varepsilon)}\right) \leq \varepsilon+\sum_{q \in \mathcal{G}} c \varepsilon \ell_{q} \\
& \leq \varepsilon+c \varepsilon \mathcal{H}^{1}\left(J_{u}\right)
\end{aligned}
$$

and, since by Proposition 4.9 the total length of $J_{w} \cap \hat{q}^{(1-\varepsilon)}$ is bounded by $2(1+\varepsilon) \ell_{q}$ and by $(4.9) \mathcal{H}^{1}\left(J_{u} \cap \hat{q}\right) \geq 2(1-\varepsilon) \ell_{q}$,

$$
\mathcal{H}^{1}\left(J_{w} \cap \cup_{q \in \mathcal{G}} \hat{q}^{(1-\varepsilon)}\right) \leq \sum_{q \in \mathcal{G}} 2(1+\varepsilon) \ell_{q} \leq(1+3 \varepsilon) \mathcal{H}^{1}\left(J_{u} \cap \cup_{q \in \mathcal{G}} q\right) .
$$

For the gradient term we obtain, again using Proposition 4.9 and (4.9),

$$
\begin{aligned}
\|\nabla w\|_{L^{p}(\Omega)}^{p} & \leq\|\nabla u\|_{L^{p}(\Omega)}^{p}+\sum_{q \in \mathcal{G}} \frac{c}{\varepsilon^{p}}\|\nabla u\|_{L^{p}(q)}^{p} \\
& \leq\|\nabla u\|_{L^{p}(\Omega)}^{p}+\frac{c}{\varepsilon^{p}} \sum_{q \in \mathcal{G}} \varepsilon^{p+1} \ell_{q} \leq\|\nabla u\|_{L^{p}(\Omega)}^{p}+c \varepsilon \mathcal{H}^{1}\left(J_{u}\right) .
\end{aligned}
$$

We also estimate, using the same bound and Lemma 4.4 in each square,

$$
\|w-u\|_{L^{p}(\Omega)}^{p} \leq \sum_{q \in \mathcal{G}} c \ell_{q}^{p}\|\nabla u-\nabla w\|_{L^{p}(q)}^{p} \leq \sum_{q \in \mathcal{G}} c \varepsilon^{p+1} \ell_{q}^{p+1} \leq c \varepsilon^{p+1}|\Omega|(\operatorname{diam} \Omega)^{p-1} .
$$

This concludes the treatment of the main part of the jump set.

We summarize what we have obtained so far. Given $\varepsilon>0$, we obtained a finite set of squares $\mathcal{G}$ and $w \in S B V_{y}^{p}(\Omega)$ such that $J_{w} \cap \hat{q}^{(1-\varepsilon)}$ consists of two segments for any $q \in \mathcal{G},\|\nabla w\|_{L^{p}(\Omega)} \leq\|\nabla u\|_{L^{p}(\Omega)}+M_{u} \varepsilon, \mathcal{H}^{1}\left(J_{w} \cap \Omega\right) \leq \mathcal{H}^{1}\left(J_{u} \cap \Omega\right)+M_{u} \varepsilon$, $u=w$ around $\partial \Omega,\|u-w\|_{L^{1}(\Omega)} \leq M_{u} \varepsilon$, and $\mathcal{H}^{1}\left(J_{u} \backslash \omega\right) \leq M_{u} \varepsilon$. Here $M_{u}$ is a constant that may depend on $u$ and $\Omega$ but not on $\varepsilon$ and we let $\omega:=\cup_{q \in \mathcal{G}} \hat{q}^{(1-\varepsilon)}$ be the union of the smaller squares.

Step 2: Treatment of the small part of the jump set.

We intend to cover $\Omega \backslash \omega$ with squares much smaller than those composing $\omega$. We fix $\delta>0$, chosen below. We choose $N$ families of squares $\mathcal{F}_{1}^{\prime}, \ldots, \mathcal{F}_{N}^{\prime}$ which cover $\Omega$ as in Lemma 4.10. Inside $\omega$ we do not need to modify the function $w$ any more, hence the squares in $\mathcal{G}^{\prime}:=\left\{q \in \mathcal{F}^{\prime}: q \subset \omega\right\}$ need not be touched. We set $\mathcal{F}_{k}:=\mathcal{F}_{k}^{\prime} \backslash \mathcal{G}^{\prime}$, and correspondingly $\mathcal{F}:=\mathcal{F}^{\prime} \backslash \mathcal{G}^{\prime}=\cup_{k} \mathcal{F}_{k}$. Property (i) now reads $\Omega=\omega \cup \bigcup_{q \in \mathcal{F}} q$, (ii) and (iii) still hold, the estimates on $\mathcal{N}_{k}$ are also still valid. Since $J_{w}$ is union of two segments inside each of the squares in $\mathcal{G}$, we have

$$
\mathcal{H}^{1}\left(J_{w} \cap \bigcup_{q \in \mathcal{F}} q\right) \leq \mathcal{H}^{1}\left(J_{u} \cap \Omega \backslash \omega\right)+2 \delta \# \mathcal{G} \leq 2 M_{u} \varepsilon
$$

provided $\delta$ is chosen sufficiently small (on a scale set by $\varepsilon$ and $\mathcal{G}$, which in turn depends on $\varepsilon$ and $u$ ). 
Fix $\eta \in\left(0,10^{-2}\right)$. Let $\mathcal{B}:=\left\{q \in \mathcal{F}: \mathcal{H}^{1}\left(J_{w} \cap q\right) \geq \eta \ell_{q}\right\}$ be the set of "bad" squares, on which we cannot use Proposition 4.7.

We intend to iteratively apply the constructions in the individual squares of each family. We set $z_{0}:=w$.

We first explain how to construct $z_{k}$ from $z_{k-1}$, working on the (disjoint) squares of the subfamily $\mathcal{F}_{k}$. We describe the procedure at step $k$, dropping the index from the notation for simplicity. We set $y_{0}:=z_{k-1}$, let $\left(q_{m}\right)_{m \in \mathbb{N}}$ be an enumeration of $\mathcal{F}_{k}$, and define for $m \in \mathbb{N}$ the function $y_{m}$ by $y_{m}=y_{m-1}$ on $\Omega \backslash q_{m}$, and inside $q_{m}$ as the result of Proposition 4.7 with $\rho:=\eta^{1 / 2} \ell_{q_{m}}$ if $q_{m} \notin \mathcal{B}$, and the result of Proposition 4.8 if $q_{m} \in \mathcal{B}$. We obtain

$$
\mathcal{H}^{1}\left(J_{y_{m}}\right) \leq \mathcal{H}^{1}\left(J_{y_{m-1}}\right)+c \mathcal{H}^{1}\left(J_{y_{m-1}} \cap q_{m}\right) .
$$

Since $y_{m-1}=y_{0}$ in $q_{m}$ and the squares are disjoint, this gives $\mathcal{H}^{1}\left(J_{y_{m}}\right) \leq(1+$ c) $\mathcal{H}^{1}\left(J_{y_{0}}\right)$ independently of $m$. Further, the constructions give

$$
\left|D y_{m}-D y_{m-1}\right|(\Omega)=\left|D y_{m}-D y_{m-1}\right|\left(q_{m}\right) \leq c\left|D y_{m-1}\right|\left(q_{m}\right)=c\left|D y_{0}\right|\left(q_{m}\right),
$$

where as above we used that $y_{m-1}=y_{0}$ on $q_{m}$. Since the $q_{m}$ are disjoint, this shows that $\sum_{m}\left|D y_{m}-D y_{m-1}\right|(\Omega) \leq c\left|D y_{0}\right|(\Omega)<\infty$, hence (recalling $y_{m}=y_{0}$ on $\partial \Omega$ ) the sequence $y_{m}$ is a Cauchy sequence in $B V$. Let $y_{\infty}$ be the limit. Since all $y_{m}$ have the same traces on $\partial \Omega$ as $y_{0}$, so does $y_{\infty}$. At the same time, since $\eta \leq \eta^{1 / p^{\prime}}$

$\left\|\nabla y_{m}\right\|_{L^{p}\left(q_{m}\right)}^{p} \leq\left\|\nabla y_{m-1}\right\|_{L^{p}\left(q_{m}\right)}^{p}+c \eta^{p /\left(2 p^{\prime}\right)}\left\|\nabla y_{m-1}\right\|_{L^{p}\left(q_{m}\right)}^{p}+c\left\|\nabla y_{m-1}\right\|_{L^{p}\left(q_{m}\right)}^{p} \chi_{q_{m} \in \mathcal{B}}$

where the last term only appears if $q_{m}$ is in $\mathcal{B}$. Again, since $y_{m-1}=y_{0}$ on $q_{m}$ and the squares are disjoint we obtain a uniform bound on $\left\|\nabla y_{m}\right\|_{L^{p}(\Omega)}$,

$$
\left\|\nabla y_{m}\right\|_{L^{p}(\Omega)}^{p} \leq\left(1+c \eta^{p /\left(2 p^{\prime}\right)}\right)\left\|\nabla y_{0}\right\|_{L^{p}(\Omega)}^{p}+c \sum_{q \in \mathcal{F}_{k} \cap \mathcal{B}}\left\|\nabla y_{0}\right\|_{L^{p}(q)}^{p} .
$$

By the $S B V^{p}$ compactness theorem, the limit $y_{\infty}$ belongs to $S B V^{p}(\Omega)$. We define $z_{k}$ as $y_{\infty}$. It is clear that $z_{k} \in S B V_{y}^{p}(\Omega)$ with $z_{k}=u$ on $\partial \Omega$. Further, using Lemma 4.4

$$
\left\|z_{k}-z_{k-1}\right\|_{L^{p}(\Omega)}^{p} \leq \sum_{m} c \ell_{q_{m}}^{p}\left\|\nabla y_{m}\right\|_{L^{p}\left(q_{m}\right)}^{p} \leq c \delta^{p}\left\|\nabla z_{k-1}\right\|_{L^{p}\left(q_{m}\right)}^{p} .
$$

Iterating this procedure over the $N$ families we obtain the function $z_{N}$. By construction, $z_{N} \in S B V_{y}^{p}(\Omega)$ and $z_{N}=u$ on $\partial \Omega$. Further, any open set $\hat{\Omega} \subset \subset \Omega$ intersects only finitely many squares, therefore $J_{z_{N}} \cap \hat{\Omega}$ is a finite union of segments. It remains to estimate the norms. We first observe that, with $\Omega^{\prime}:=\cup_{q \in \mathcal{F}}$,

$$
\mathcal{H}^{1}\left(J_{z_{k}} \cap \Omega^{\prime}\right) \leq c \mathcal{H}^{1}\left(J_{z_{k-1}} \cap \Omega^{\prime}\right)
$$


which immediately gives $\mathcal{H}^{1}\left(J_{z_{N}} \cap \Omega^{\prime}\right) \leq c^{N} \mathcal{H}^{1}\left(J_{w} \cap \Omega^{\prime}\right) \leq 2 c^{N} M_{u} \varepsilon$. Further, by $(4.11)$

$$
\left\|\nabla z_{k}\right\|_{L^{p}(\Omega)}^{p} \leq\left(1+c \eta^{p /\left(2 p^{\prime}\right)}\right)\left\|\nabla z_{k-1}\right\|_{L^{p}(\Omega)}^{p}+c \sum_{q \in \mathcal{F}_{k} \cap \mathcal{B}}\left\|\nabla z_{k-1}\right\|_{L^{p}(q)}^{p} .
$$

In order to estimate the last term, we use the second part of Lemma 4.10. The key property of the construction we use here is the fact that at each step $k$ the function is only modified in the squares of $\mathcal{F}_{k}$, which are disjoint. Let now $q \in \mathcal{F}$ be a generic square. Since only the changes in squares $q^{\prime} \in \mathcal{F}_{k}$ which intersect $q$ modify the function inside $q$, we obtain from (4.10)

$$
\left\|\nabla z_{k}\right\|_{L^{p}(q)} \leq c \sum_{q^{\prime} \in \mathcal{F}_{k} \cap \mathcal{N}_{1}(q)}\left\|\nabla z_{k-1}\right\|_{L^{p}\left(q^{\prime}\right)} .
$$

Iterating this condition, and recalling the properties in Lemma 4.10, we have

$$
\left\|\nabla z_{k}\right\|_{L^{p}(q)} \leq c^{k} \sum_{q^{\prime} \in \mathcal{N}_{k}(q)}\|\nabla w\|_{L^{p}\left(q^{\prime}\right)} .
$$

Since any square $q^{\prime}$ can be reached only starting from its $N$-neighbours, the combinatorial coefficient is uniformly bounded. We conclude that

$$
\sum_{k} \sum_{q \in \mathcal{F}_{k} \cap \mathcal{B}}\left\|\nabla z_{k-1}\right\|_{L^{p}(q)} \leq c\|\nabla w\|_{L^{p}(\hat{\omega})}
$$

where

$$
\hat{\omega}:=\bigcup_{q \in \mathcal{F} \cap \mathcal{B}} \bigcup_{q^{\prime} \in \mathcal{N}_{N}(q)} q^{\prime} .
$$

It remains to estimate the size of the set $\hat{\omega}$. Since $\ell_{q^{\prime}} \leq a^{N} \ell_{q}$, we have

$$
\begin{aligned}
|\hat{\omega}| & \leq 4 \sum_{q \in \mathcal{F} \cap \mathcal{B}}\left(a^{2 N} \ell_{q}^{2}\right) \# \mathcal{N}_{N}(q) \leq 4 a^{2 N} b^{N} \sum_{q \in \mathcal{F} \cap \mathcal{B}} \ell_{q}^{2} \\
& \leq 4 \frac{a^{2 N} b^{N}}{\eta} \sum_{q \in \mathcal{F} \cap \mathcal{B}} \delta \mathcal{H}^{1}\left(J_{w} \cap q\right) \leq 4 \frac{a^{2 N} b^{N} \delta}{\eta} \mathcal{H}^{1}\left(J_{w} \cap \Omega^{\prime}\right) \leq \frac{8 M_{u} a^{2 N} b^{N} \varepsilon \delta}{\eta} .
\end{aligned}
$$

At this point we choose $\eta:=\varepsilon$. In the limit $\delta \rightarrow 0$ we have $|\hat{\omega}| \rightarrow 0$, therefore if $\delta$ is sufficiently small we have $\|\nabla w\|_{L^{p}(\hat{\omega})} \leq \varepsilon$, and so (4.13) yields $\left\|\nabla z_{N}\right\|_{L^{p}(\Omega)}^{p} \leq$ $\left(1+C \varepsilon^{p /\left(2 p^{\prime}\right)}\right)\|\nabla w\|_{L^{p}(\Omega)}^{p}+\varepsilon$. Further, for $\delta$ sufficiently small iterating (4.12) for $k=1, \ldots, N$ yields $\left\|z_{N}-w\right\|_{L^{p}} \leq \varepsilon$.

Finally, we define $v_{j}$ as the function $z_{N}$ obtained with $\varepsilon:=1 / j$.

Step 3: Inclusion of the condition on $U$.

If there are $U \subset \Omega$ open and $R>0$ such that $J_{u} \cap B_{R}(U)=\emptyset$ we modify the construction slightly. Let first the maximal diameter of all squares (in both iterations) be smaller then $R / 2$ (this means $2 \sqrt{2} \ell_{q}<R / 2$ for all squares $q$ ). 
Further, at the beginning of Step 2 we also exclude all squares which contain no jump set (and in which, therefore, no action is needed). Precisely, we replace $\mathcal{G}^{\prime}$ by $\mathcal{G}^{\prime \prime}:=\left\{q \in \mathcal{F}^{\prime}: q \subset \omega\right.$ or $\left.\mathcal{H}^{1}\left(q \cap J_{w}\right)=0\right\}$. Then none of the "surviving" squares touches $U$, hence $u$ is not modified in $U$. All other properties still hold.

\section{Acknowledgments}

We thank Hans Knüpfer for helpful discussions. This work was partially supported by the Deutsche Forschungsgemeinschaft through the Sonderforschungsbereich 1060 "The mathematics of emergent effects", project A6.

\section{A Scaling Law}

In this appendix we prove Theorem 1.1. We follow the paths of the proof of the scaling law for the special case $p=2$ (see $[35,36,16,23,45]$ ).

Proof of Theorem 1.1. Step 1: Upper bound

Note that the constant function $u_{c}:=0$ yields $I_{\theta, \varepsilon}^{p}\left(u_{c}\right)=\theta^{p}$. If $\varepsilon \leq \theta^{p}$ we construct a test function $u_{b}$ with $I_{\theta, \varepsilon}^{p}\left(u_{b}\right) \leq c \theta^{p}\left(\varepsilon / \theta^{p}\right)^{p /(p+1)}$, using the variant of the branching construction from [36] given in [45] in the formulation of [18]. The construction given in [18, Lemma 5.2] shows that for arbitrary $\ell>0$ and $h>0$ there exists a function $b=b^{(\ell, h)}:(0, \ell) \times \mathbb{R} \rightarrow \mathbb{R}$ with the following properties:

(i) $b\left(x_{1}, 0\right)=0, b\left(x_{1}, \cdot\right)$ is $h$-periodic,

$$
b\left(\ell, x_{2}\right)= \begin{cases}-\theta x_{2} & \text { if } 0 \leq x_{2} \leq h(1-\theta) / 2 \\ (1-\theta)\left(x_{2}-h / 2\right) & \text { if } h(1-\theta)) / 2 \leq x_{2} \leq h(1+\theta) / 2, \\ -\theta x_{2}+\theta h & \text { if } h(1+\theta) / 2 \leq x_{2} \leq h\end{cases}
$$

(ii) $b\left(0, x_{2}\right)=\frac{1}{2} b\left(\ell, 2 x_{2}\right)$,

(iii) $\left\|\partial_{1} b\right\|_{L^{p}((0, \ell) \times(0, h))} \leq c \frac{\theta^{p} h^{p+1}}{\ell^{p-1}}$,

(iv) $\int_{(0, \ell) \times(0, h)}\left|D^{2} b\right| \leq C(\ell+\theta h)$,

(v) $\partial_{2} b \in\{-\theta, 1-\theta\}$ almost everywhere.

We now proceed as in [36, Lemma 1]. We choose a refining parameter $\alpha \in$ $\left(2^{-p /(p-1)}, 2^{-1}\right)$, and choose $N \in \mathbb{N}$ such that $N \sim\left(\theta^{p} / \varepsilon\right)^{1 /(p+1)}$. We decompose $(0,1) \times(0,1)$ into rectangles

$$
R_{i j}:=\left(\alpha^{i+1}, \alpha^{i}\right) \times\left(\frac{j}{2^{i} N}, \frac{j+1}{2^{i} N}\right), \quad i=0,1, \ldots \text { and } j=0, \ldots, 2^{i} N-1 .
$$

For $i \leq I$ with $(2 \alpha)^{I} \sim \theta / N$, we set $u_{b}\left(x_{1}, x_{2}\right)=b^{\left(\ell_{i}, h_{i}\right)}\left(x_{1}-\alpha^{i+1}, x_{2}\right)$ on $R_{i 0}$, where $\ell_{i}:=(1-\alpha) \alpha^{i}$ and $h_{i}:=1 /\left(2^{i} N\right)$. The function $u_{b}$ is then extended 
$1 /\left(2^{i} N\right)$-periodically in $x_{2}$-direction to the remaining $R_{i j}$. Finally, we use linear interpolation in $x_{1}$ on $\left(0, \alpha^{I+1}\right) \times(0,1)$. The total energy in $\left(\alpha^{I+1}, 1\right) \times(0,1)$ is then estimated by

$$
C \sum_{i=1}^{I}\left(\frac{\theta^{p}}{\left(2^{i} N\right)^{p+1} \alpha^{i(p-1)}} 2^{i} N+\varepsilon \alpha^{i} 2^{i} N\right) \leq C(\varepsilon \theta)^{p /(p+1)} .
$$

Since $\alpha<(2 \alpha)^{p}, \alpha^{I} \leq(2 \alpha)^{p I} \sim(\theta / N)^{p} \sim(\varepsilon \theta)^{p /(p+1)}$ and the transition layer obeys the analogue upper bound.

Step 2: Lower bound

To derive the lower bound, we follow closely the lines of [45, Proof of Theorem 1], which in turn is based on [16]. Let $\theta, \varepsilon, p$ be given as in Theorem 1.1, and fix $u \in \mathcal{B}$ arbitrary. Set $t:=\min \left\{1,\left(\varepsilon / \theta^{p}\right)^{1 /(p+1)}\right\}$. Choose $J:=[y, y+t] \subset(0,1)$ such that

$I_{J}(u):=\int_{(0,1) \times J}\left|\partial_{1} u\right|^{p}+\min \left\{\left|\partial_{2} u+\theta\right|^{p},\left|\partial_{2} u-(1-\theta)\right|^{p}\right\} \mathrm{d} x+\varepsilon\left|D^{2} u\right|((0,1) \times J) \leq 2 t I_{\theta, \varepsilon}^{p}(u)$.

By Fubini, there exists $M \subset(0,1)$ with $\mathcal{L}^{1}(M)>0$ such that for all $x_{1} \in M$

$$
\begin{aligned}
& \int_{\left\{x_{1}\right\} \times J}\left|\partial_{1} u\right|^{p}+\min \left\{\left|\partial_{2} u+\theta\right|^{p},\left|\partial_{2} u-(1-\theta)\right|^{p}\right\} \mathrm{d} x_{2} \\
& \leq 3 \int_{(0,1) \times J}\left|\partial_{1} u\right|^{p}+\min \left\{\left|\partial_{2} u+\theta\right|^{p},\left|\partial_{2} u-(1-\theta)\right|^{p}\right\} \mathrm{d} x
\end{aligned}
$$

and

$$
\left|\partial_{2} \partial_{2} u\right|\left(\left\{x_{1}\right\} \times J\right) \leq 3\left|D^{2} v\right|((0,1) \times J) .
$$

We decompose $M:=M_{1} \cup M_{2} \cup M_{3}$, where

$$
\begin{aligned}
& M_{1}:=\left\{x_{1} \in M:\left|\partial_{2} u+\theta\right| \leq\left|\partial_{2} u-(1-\theta)\right| \text { a.e. } x_{2} \in J\right\}, \\
& M_{2}:=\left\{x_{1} \in M:\left|\partial_{2} u+\theta\right| \geq\left|\partial_{2} u-(1-\theta)\right| \text { a.e. } x_{2} \in J\right\}, \\
& M_{3}:=M \backslash\left(M_{1} \cup M_{2}\right) .
\end{aligned}
$$

One of the three sets has positive measure. If $M_{1}$ has positive measure, then fix $x_{1} \in M_{1}$. We use the following variant of an estimate from [16, Lemma 1] (see [45])

$$
t^{2} \theta \lesssim \min _{c \in \mathbb{R}}\left\|u\left(x_{1}, x_{2}\right)+\theta x_{2}-c\right\|_{L^{1}(J)}+\left\|u\left(x_{1}, \cdot\right)\right\|_{L^{1}(J)} .
$$

By Poincaré's inequality and definition of $M_{1}$ (recall that $p^{\prime}:=p /(p-1)$ ),

$$
\min _{c \in \mathbb{R}}\left\|u\left(x_{1}, x_{2}\right)+\theta x_{2}-c\right\|_{L^{1}(J)} \leq t\left\|\partial_{2} u+\theta\right\|_{L^{1}(J)} \leq t^{1+1 / p^{\prime}}\left(I_{J}(u)\right)^{1 / p},
$$

and by the boundary conditions and the fundamental theorem of calculus,

$$
\left\|u\left(x_{1}, \cdot\right)\right\|_{L^{1}(J)} \leq\left\|\partial_{1} u\right\|_{L^{1}((0,1) \times J)} \leq t^{1 / p^{\prime}}\left(I_{J}(u)\right)^{1 / p} .
$$


Therefore, in this case,

$$
I_{\theta, \varepsilon}^{p}(u) \gtrsim \theta^{p} \min \left\{t^{p}, 1\right\} \geq \theta^{p} \min \left\{1,\left(\varepsilon / \theta^{p}\right)^{p /(p+1)}\right\} .
$$

Similarly, if $M_{2}$ has positive measure, we obtain the larger lower bound $I(u) \gtrsim$ $\min \left\{t^{p}, 1\right\}$. Finally, if $M_{3}$ has positive measure, fix $x \in M_{3}$. There are two possibilities: Either

$$
\min \left\{\left|\partial_{2} u+\theta\right|,\left|\partial_{2} u-(1-\theta)\right|\right\} \geq 1 / 4 \quad \text { for a.e. } x_{2} \in J,
$$

which implies

$$
\frac{t}{4^{p}} \leq \int_{\left\{x_{1}\right\} \times J} \min \left\{\left|\partial_{2} v+\theta\right|^{p},\left|\partial_{2} v-(1-\theta)\right|^{p}\right\} \mathrm{d} x_{2} \leq I_{J}(u),
$$

or $\left|\partial_{2} \partial_{2} u\right|\left(\left\{x_{1}\right\} \times J\right) \geq 1 / 4$. Hence,

$$
I_{\theta, \varepsilon}^{p}(u) \gtrsim \min \{\varepsilon / t, 1\} \gtrsim \theta^{p} \min \left\{\left(\varepsilon / \theta^{p}\right)^{p /(p+1)}, 1\right\} .
$$

\section{References}

[1] L. Ambrosio, N. Fusco, And D. Pallara, Functions of bounded variation and free discontinuity problems, Oxford Mathematical Monographs, Oxford University Press, New York, 2000.

[2] C. Bechtold, C. Chluba, R. Lima de Miranda, and E. Quandt, High cyclic stability of the elastocaloric effect in sputtered TiNiCu shape memory films, Applied Physics Letters, 101 (2012), p. 091903.

[3] P. Bella And R. V. Kohn, Wrinkles as the result of compressive stresses in an annular thin film, Comm. on Pure and Appl. Math., 67 (2014), pp. 693747 .

[4] H. Ben Belgacem, S. Conti, A. DeSimone, and S. Müller, Rigorous bounds for the Föppl-von Kármán theory of isotropically compressed plates, J. Nonlinear Sci., 10 (2000), pp. 661-683.

[5] H. Ben Belgacem, S. Conti, A. DeSimone, and S. Müller, Energy scaling of compressed elastic films, Arch. Rat. Mech. Anal., 164 (2002), pp. 137.

[6] A. Capella and F. Otto, A rigidity result for a perturbation of the geometrically linear three-well problem, Comm. Pure Appl. Math., 62 (2009), pp. 1632-1669. 
[7] —, A quantitative rigidity result for the cubic-to-tetragonal phase transition in the geometrically linear theory with interfacial energy, Proc. Roy. Soc. Edinburgh Sect. A, 142 (2012), pp. 273-327.

[8] A. Chan, Energieskalierung, Gebietsverzweigung und SO(2)-Invarianz in einem fest-fest Phasenübergangsproblem, PhD thesis, Bonn University, 2013.

[9] A. Chan And S. Conti, Energy scaling and domain branching in solidsolid phase transitions, in Singular Phenomena and Scaling in Mathematical Models, M. Griebel, ed., Springer International Publishing, 2014, pp. 243260 .

[10] — Energy scaling and branched microstructures in a model for shapememory alloys with SO(2) invariance, Math. Models Methods App. Sci., 25 (2015), pp. 1091-1124.

[11] C. Chluba, W. Ge, R. Lima de Miranda, J. Strobel, L. Kienle, E. QuANDT, AND M. Wuttig, Ultralow-fatigue shape memory alloy films, Science, 348 (2015), pp. 1004-1007.

[12] R. Choksi, S. Conti, R. Kohn, And F. Otтo, Ground state energy scaling laws during the onset and destruction of the intermediate state in a type I superconductor, Comm. Pure Appl. Math., 61(5) (2008), pp. 595-626.

[13] R. Choksi, R. Kohn, And F. Отto, Domain branching in uniaxial ferromagnets: a scaling law for the minimum energy, Comm. Math. Phys., 201(1) (1998), pp. 61-79.

[14] — Energy minimization and flux domain structure in the intermediate state of a type-I superconductor, J. Nonlin. Sc., 14(2) (2004), pp. 119-171.

[15] R. Choksi And R. V. Kohn, Bounds on the micromagnetic energy of a uniaxial ferromagnet, Comm. Pure Appl. Math., 51 (1998), pp. 259-289.

[16] S. ConTI, A lower bound for a variational model for pattern formation in shape-memory alloys, Cont. Mech. Thermodyn., 17 (6) (2006), pp. 469-476.

[17] S. Conti, F. Otto, And S. Serfaty, Branched microstructures in the Ginzburg-Landau model of type-I-superconductors. arxiv:1507.00836v1, 2015.

[18] S. Conti And B. Zwicknagl, Low volume-fraction microstructures in martensites and crystal plasticity. arXiv:1507.04521, 2015.

[19] G. Cortesani and R. Toader, A density result in $S B V$ with respect to non-isotropic energies, Nonlinear Analysis: Theory, Methods \& Applications, 38 (1999), pp. 585-604. 
[20] J. Cui, Y. Chu, O. Famodu, Y. Furuya, J. Hattrick-Simpers, R. James, A. Ludwig, S. Thienhaus, M. Wuttig, Z. Zhang, And I. TAKEUCHI, Combinatorial search of thermoelastic shape-memory alloys with extremely small hysteresis width, Nature materials, 5 (2006), pp. 286290.

[21] E. De Giorgi, M. Carriero, and A. Leaci, Existence theorem for a minimum problem with free discontinuity set, Archive for Rational Mechanics and Analysis, 108 (1989), pp. 195-218.

[22] F. Dibos And E. SÉRÉ, An approximation result for the minimizers of the Mumford-Shah functional, Boll. Un. Mat. Ital. A (7), 11 (1997), pp. 149-162.

[23] J. DiermeieR, Nichtkonvexe Variationsprobleme und Mikrostrukturen, Bachelor's thesis, Universität Bonn, 2010.

[24] — Domain branching in linear elasticity, Master's thesis, Universität Bonn, 2013.

$[25]$ - A low volume-fraction limit for martensitic microstructures in shapememory alloys, Proc. Appl. Math. Mech., 15 (2015), pp. 541-542.

[26] —_ Analysis of microstructures in shape-memory-alloys: A low-volumefraction limit, $\mathrm{PhD}$ thesis, Bonn University, in preparation.

[27] L. Evans and R. Gariepy, Measure Theory and Fine Properties of Functions, Studies in Advanced Mathematics, CRC Press, 1992.

[28] G. Friesecke, R. D. James, And S. Müller, A theorem on geometric rigidity and the derivation of nonlinear plate theory from three-dimensional elasticity, Comm. Pure Appl. Math., 55 (2002), pp. 1461-1506.

[29] R. JAMES AND Z. ZhANG, A way to search for multiferroic materials with "unlikely" combinations of physical properties, in The Interplay of Magnetism and Structure in Functional Materials, L. Manosa, A. Planes, and A. Saxena, eds., vol. 79 of Springer Series in Materials Science, Springer, 2005.

[30] W. Jin And P. Sternberg, Energy estimates of the von Kármán model of thin-film blistering, J. Math. Phys., 42 (2001), pp. 192-199.

[31] — In-plane displacements in thin-film blistering, Proc. R. Soc. Edin. A, 132A (2002), pp. 911-930.

[32] H. Knüpfer and R. V. Kohn, Minimal energy for elastic inclusions, Proc. R. Soc. Lond. Ser. A Math. Phys. Eng. Sci., 467 (2011), pp. 695-717.

[33] H. Knüpfer, R. V. Kohn, And F. Otto, Nucleation barriers for the cubicto-tetragonal phase transformation, Comm. Pure Appl. Math., 66 (2013), pp. 867-904. 
[34] H. KnÜpfer and C. Muratov, Domain structure of bulk ferromagnetic crystals in applied fields near saturation, J. Nonlinear Sc., (2011), pp. 1-42.

[35] R. Kohn And S. MüLler, Branching of twins near an austenite-twinned martensite interface, Phil. Mag. A, 66 (1992), pp. 697-715.

[36] R. Kohn And S. MÜller, Surface energy and microstructure in coherent phase transitions, Comm. Pure Appl. Math., XLVII (1994), pp. 405-435.

[37] M. Louie, M. Kislitsyn, K. Bhattacharya, and S. Haile, Phase transformation and hysteresis behavior in $\mathrm{Cs}_{1-x} \mathrm{Rb}_{x} \mathrm{H}_{2} \mathrm{PO}_{4}$, Solid state ionics, 181 (2010), pp. 173-179.

[38] D. Mumford And J. Shah, Optimal approximations by piecewise smooth functions and associated variational problems, Comm. Pure Appl. Math., 42 (1989), pp. 577-685.

[39] F. Otto and T. Viehmann, Domain branching in uniaxial ferromagnets: asymptotic behavior of the energy, Calc. Var. PDE, 38 (2010), pp. 135-181.

[40] V. Srivastava, X. Chen, And R. James, Hysteresis and unusual magnetic properties in the singular Heusler alloy $\mathrm{Ni}_{45} \mathrm{Co}_{5} \mathrm{Mn}_{40} \mathrm{Sn}_{10}$, Appl. Phys. Lett., 97 (2010), p. 014101.

[41] E. M. Stein, Singular Integrals and differentiability properties of functions, Princeton University Press, 1970.

[42] R. Zarnetta, R. Takahashi, M. Young, A. Savan, Y. Furuya, S. Thienhaus, B. Maass, M. Rahim, J. Frenzel, H. Brunken, Y. Chu, V. Srivastava, R. James, I. Takeuchi, G. Eggeler, and A. Ludwig, Identification of quaternary shape memory alloys with near-zero thermal hysteresis and unprecedented functional stability, Advanced Functional Materials, 20(12) (2010), pp. 1917-1923.

[43] Z. Zhang, Special lattice parameters and the design of low hysteresis materials, $\mathrm{PhD}$ thesis, University of Minnesota, 2007.

[44] Z. Zhang, R.D.James, AND S. MÜLler, Energy barriers and hysteresis in martensitic phase transformations, Acta Materialia, 57(15) (2009), pp. 43324352 .

[45] B. Zwicknagl, Microstructures in low-hysteresis shape memory alloys: Scaling regimes and optimal needle shapes, Arch. Rat. Mech. Anal., 213 (2014), pp. $355-421$. 